\title{
Efficient Inference for Multilingual Neural Machine Translation
}

\author{
Alexandre Bérard* \\ NAVER LABS Europe
}

\author{
Dain Lee ${ }^{\dagger}$ \\ NAVER Corp.
}

\author{
Stéphane Clinchant ${ }^{\star}$ \\ NAVER LABS Europe
}

\author{
Kweonwoo Jung ${ }^{\ddagger}$ \\ NAVER Corp.
}

\author{
Vassilina Nikoulina ${ }^{\star}$ \\ NAVER LABS Europe
}

\begin{abstract}
Multilingual NMT has become an attractive solution for MT deployment in production. But to match bilingual quality, it comes at the cost of larger and slower models. In this work, we consider several ways to make multilingual NMT faster at inference without degrading its quality. We experiment with several "light decoder" architectures in two 20language multi-parallel settings: small-scale on TED Talks and large-scale on ParaCrawl. Our experiments demonstrate that combining a shallow decoder with vocabulary filtering leads to more than $\times 2$ faster inference with no loss in translation quality. We validate our findings with BLEU and chrF (on 380 language pairs), robustness evaluation and human evaluation.
\end{abstract}

\section{Introduction}

Multilingual machine translation (Johnson et al., 2017; Bapna and Firat, 2019; Aharoni et al., 2019; Zhang et al., 2020; Fan et al., 2020; Lyu et al., 2020) has made a lot of progress in the last years. It is attractive because it allows handling multiple language directions within a single model, thus significantly reducing training and maintenance costs. However, to preserve good performance across all the language pairs, both the vocabulary size and model size have to be increased compared to bilingual NMT, which hurts inference speed. For example, the recently released M2M-100 (Fan et al., 2020) has 15B parameters and needs multiple GPUs for inference. The problem of inference speed has been well studied in bilingual settings (Kasai et al., 2021a,b; Chen et al., 2018; Hsu et al., 2020; Kim et al., 2019; Li et al., 2020; Shi and Knight, 2017). One line of work consists in using lighter decoder architectures (e.g., shallow decoder: Kasai et al., 2021a, RNN decoder: Chen

\footnotetext{
* first. lastenaverlabs. com

† dain.lenavercorp.com

${ }^{\ddagger}$ kweonwoo. jung@navercorp. com
}

et al., 2018; Kim et al., 2019; Lei, 2021). These works demonstrate that it is possible to significantly speed up inference with almost no loss in translation quality as measured by BLEU.

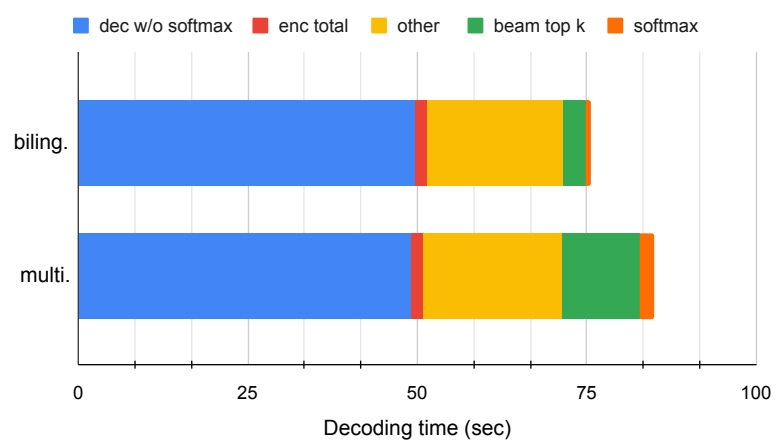

Figure 1: Decoding time spent in different components of bilingual (vocab size 16k) vs multilingual (vocab size 69k) Transformer Big 6-6 models on TED2020 valid EN-DE (average over 10 runs).

Figure 1 compares the inference time spent in each NMT component by bilingual and multilingual models of the same architecture but with different vocabulary sizes. The decoder is also the bottleneck in the multilingual model which suggests that we can expect similar speed gains with a lighter decoder. It also indicates that some speed gain could be obtained by reducing vocabulary size (which impacts both beam search and softmax).

However, it is not so obvious that lighter decoder architectures would preserve translation quality in multilingual settings, where the decoder may need more capacity to deal with multiple languages. Therefore, the goal of this work is to benchmark different architectures in terms of inference speed/translation quality trade-off and to identify the best combination for multilingual NMT. The contributions of this paper are:

- A benchmark of two popular "light decoder" NMT architectures (deep encoder / shallow decoder, Kasai et al., 2021a; and RNN decoder, Chen et al., 2018) on two multilingual datasets 
(TED Talks and ParaCrawl) in both Englishcentric and multi-parallel settings. It demonstrates that the previous findings transfer to multilingual models.

- A combination of shallow decoder with perlanguage vocabulary filtering for further speed gains (achieving a global 2 to $3 \times$ speed-up over the baseline) with no loss in translation quality.

- Experiments with separate language-specific shallow decoders, which trade memory for higher BLEU performance, with comparable speed as the single-decoder approach.

- A validation of these findings through extensive analysis, including robustness evaluation and human evaluation.

\section{Related work}

Lightweight decoder. As shown in Figure 1, more than half of the inference time is devoted to the decoder and $30 \times$ more time is spent in the decoder than in the encoder (due to the autoregressive nature of the models). This explains why many efficient NMT works focus on lightweight alternatives to the Transformer decoder. Kim et al. (2019) perform an extensive study of various lightweight RNN architectures and obtain a $4 \times$ gain in inference speed. Kasai et al. (2021a) show that, in bilingual settings, Transformer models with a deep encoder and shallow decoder (e.g., 10-2) can achieve similar BLEU performance as baseline 6-6 Transformers, while being much faster at inference time (on a par with current non-autoregressive MT approaches). Behnke and Heafield (2020) show that it is possible to prune up to $75 \%$ of the attention heads in a Transformer, thus increasing inference speed by $50 \%$. Similarly, Hsu et al. (2020) reduce the cost of cross-attention and self-attention by replacing it with an RNN or by pruning attention heads, obtaining up to $35 \%$ higher speed.

Although most of the above works report speed improvements with similar BLEU scores as Transformer baselines, it is uncertain that the same will hold in multilingual many-to-many settings, where the decoder may need more capacity to deal with multiple languages. In particular, Kong et al. (2021) observe that single shallow decoders degrade oneto-many MT quality and propose to train shallow language-specific decoders or decoders that are specific to a language family or group of languages.

Modular multilingual NMT. Lyu et al. (2020); Escolano et al. (2021) propose modular MT models with jointly trained language-specific encoders and decoders. Such models have higher per-language capacity, increasing their performance without hurting inference speed (contrary to the common approach of training bigger multilingual models). They are also more flexible for adding new languages. Zhang et al. (2021) study how languagespecific and language-independent parameters naturally emerge in multilingual NMT. Their findings indicate that language-independent parameters can be distributed within the encoder and decoder and benefit final NMT performance.

\section{Inference speed-up methods}

\subsection{Deep encoder, shallow decoder}

First, we analyze how deep encoder / shallow decoder models Kasai et al. (2021a) behave in multilingual settings (many-to-many English-centric and multi-parallel).

Our initial experiments in bilingual settings showed that a 12-2 architecture gives the best BLEU/speed trade-off (also reported by Li et al., 2021). We thus focus on this architecture and compare it with 6-6 and 6-2 architectures.

We find that in some cases (with Transformer Base on TED Talks), post-norm 12-2 models ${ }^{1}$ fail to converge when trained from scratch. When this happens, we initialize the 12-2 model with a pretrained 6-6 model's parameters, by duplicating its encoder layers and taking its bottom 2 decoder layers. See Table 9 in Appendix for a comparison between this approach, training from scratch, and pre-norm Transformers.

\subsection{RNN decoder}

Chen et al. (2018) first introduced a hybrid model combining a Transformer encoder with an RNN decoder. Hybrid Transformer/RNN models are considered a good practical choice in production settings due to their ideal performance-speed tradeoff (Caswell and Bowen Liang, 2020). However, Chen et al. (2018) do not experiment with hybrid models in a multilingual setting, nor do they try shallower RNN decoders. We experiment with 12layer Transformer encoders combined with either 2-layer or 3-layer LSTM decoders (noted Hybrid 12-2 / 12-3). ${ }^{2}$ Because LSTMs are slower to train,

\footnotetext{
${ }^{1}$ We prefer post-norm Transformers, as Liu et al. (2020) show that when they do converge, they often lead to better performance than pre-norm.

${ }^{2}$ Hybrid $12-3$ has a similar amount of parameters as Transformer 12-2.
} 
we first train 12-2 Transformers which we fine-tune into Hybrid models (by initializing the LSTM decoder at random). Precise architecture details are given in Appendix A.2.

\subsection{Target vocabulary filtering}

As illustrated by Figure 1, decoding speed can also be impacted by the size of the target vocabulary, because the softmax layer's complexity is linear with respect to the vocabulary size. Some solutions have been proposed to compress vocabulary in bilingual settings: vocabulary hashing or vocabulary shortlists (Shu and Nakayama, 2017; Shi and Knight, 2017; Senellart et al., 2018; Kim et al., 2019). Ding et al. (2019) also showed that the BPE size can be reduced drastically without hurting BLEU. However, reducing the BPE size too aggressively will result in longer sequences and hurt decoding speed. Lyu et al. (2020) train a separate smaller BPE model per language. However, we think that this may hurt transfer learning between languages that share words (one of the reasons why multilingual NMT uses shared vocabularies in the first place). Therefore, we propose a solution that combines the best of both worlds: have a large shared BPE vocabulary at train time, which we decompose into smaller language-specific vocabularies at test time, based on per-language token frequencies. More precisely, we train a shared BPE model of size $64 \mathrm{k}$, then for each language:

1. We tokenize its training data and count the wordpiece and character occurrences.

2. We build a vocabulary containing only tokens whose frequency is above threshold $K$ and only the $N$ most frequent wordpieces.

3. At test time, we can filter the model's target vocabulary and embeddings to only contain these tokens, resulting in a model with a single shared source embedding matrix and several smaller per-language target embedding matrices. We call this approach "test-time BPE filtering" (with parameters $N_{\text {test }}$ and $K_{\text {test }}$ ). Appendix Tables 16 $\& 17$ give the incurring parameter cost.

4. We also try combining this approach with "traintime BPE filtering" (with parameters $N_{\text {train }}$ and $\left.K_{\text {train }}\right)$. For target-side training sentences in this language, we force the shared BPE model to only generate wordpieces that belong to this language's filtered vocabulary. ${ }^{3}$

\footnotetext{
${ }^{3}$ By using subword-nmt's --vocabulary-
}

\subsection{Shared encoder, language-specific decoders}

Lyu et al. (2020) show that one can significantly increase the capacity (and thus performance) of a multilingual model without hurting decoding speed by training language-specific encoders and decoders (i.e., trading away memory for speed). We take the approach of a deeper shared encoder and multiple language-specific shallow decoders (similar to Kong et al., 2021). This approach keeps the memory usage to a reasonable value, ${ }^{4}$ and can maximize transfer learning on the encoder side.

Contrary to Lyu et al. (2020) and Kong et al. (2021), to save computation time, we first train shared multilingual MT models, which we use as initialization to our multi-decoder models (i.e., the same 2-layer decoder is copied). We use languagespecific target embeddings that are initialized with the shared embeddings obtained with the "traintime BPE filtering" technique described in the previous section. We refer to the models with shallow language-specific decoders as "multi-decoder models."

\subsection{Incremental multilingual training}

Incremental training consists in adding new languages to the model without having to retrain it on the existing languages. We measure the incremental-training ability of our single shallow decoder and language-specific shallow decoders, by applying the same technique as Berard (2021).

For a new source language, we only train a new source embedding matrix while freezing all the model's parameters. Because we substitute the shared vocabulary with a new monolingual vocabulary and keep the initial embeddings for known languages, performance on those is preserved.

When adding a new target language, we train a new shallow decoder and target embeddings for this language, while freezing the encoder parameters (similar to Lyu et al., 2020). We initialize the new decoder's parameters with those of the single decoder, or closest language-specific decoder in the multi-decoder case (e.g., Russian is initialized with Bulgarian and Latvian with Lithuanian). Contrary to Lyu et al. (2020), all our models (including the multi-decoder ones) have source-side

\footnotetext{
threshold option.

${ }^{4}$ As shown in Appendix (Table 17) a 20-language Big 12-2 multi-decoder model has $823 \mathrm{M}$ parameters in total, while a Big 6-6 or Big 12-2 multi-encoder + multi-decoder model would have $\approx 20 \times 180 \mathrm{M}=3.6 \mathrm{~B}$ parameters.
} 
language codes. So, we also train a new language code for the new target language by appending it to the source vocabulary and training its embedding while freezing all the other embeddings.

The new source and target embedding matrices are obtained by training a monolingual BPE model of size $8 \mathrm{k}$ on the new language, and initializing the embeddings of the known tokens with those from the pre-trained model's embedding matrix.

\subsection{Summary of notations}

- Base/Big 6-6/12-2 correspond respectively to Transformer Base/Big with 6 encoder layers and 6 decoder layers (resp. 12 and 2 layers).

- By default, models are trained on Englishcentric data (i.e., data in all languages paired with English, in both directions).

- Multi-parallel models are fine-tuned on data in all language directions (not just paired with English).

- Some models use test-time BPE filtering ( $N_{\text {test }}$ or $\left.K_{\text {test }}\right)$ while others use both train-time and test-time filtering ( $N_{\text {train }}$ or $\left.K_{\text {train }}\right)$.

- Hybrid models have a Transformer encoder and LSTM decoder and are fine-tuned from Englishcentric Transformers with multi-parallel data.

- Multi-decoder models have language-specific shallow decoders and are fine-tuned from English-centric models with multi-parallel data.

\section{TED Talks Experiments}

\subsection{Data and hyper-parameters}

We experiment with the TED Talks corpus (Qi et al., 2018) with the same set of 20 languages as Philip et al. (2020). ${ }^{5}$ This corpus is multi-parallel, i.e., it has training data for all $380(20 \times 19)$ language pairs (see Table 8 in Appendix for detailed statistics). It also includes official valid and test splits for all these language pairs.

We train the English-centric models for 120 epochs ( $\approx 1.8 \mathrm{M}$ updates). The Base 12-2 Englishcentric model is initialized from Base 6-6 at epoch 60 and trained for another 60 epochs, using the procedure described in Section 3.1. These models are then fine-tuned with multi-parallel data for another 10 epochs $(\approx 1.4 \mathrm{M} \text { updates })^{6}$ into single-decoder

\footnotetext{
${ }^{5}$ en, ar, he, ru, ko, it, ja, zh_cn, es, fr, pt_br, nl, tr, ro, pl, bg, vi, de, fa, hu

${ }^{6}$ Note that an "epoch" when using multi-parallel data corresponds to approximately 9 English-centric epochs in terms of updates.
}

Transformers or Hybrid models or multi-decoder Transformers. We create a shared BPE model with 64k merge operations (vocabulary size 70k) and with inline casing (Berard et al., 2019). More hyperparameters are given in Appendix A.2.

\subsection{Evaluation settings}

The TED Talks models are evaluated on the provided multi-parallel validation and test sets. Since those are already word-tokenized, we run SacreBLEU with the --tok none option. ${ }^{7}$

We report average test BLEU scores into English ( $\rightarrow \mathrm{EN}, 19$ directions), from English $(\leftarrow \mathrm{EN}$, 19 directions) and outside of English (/ EN, 342 directions). We also compute the decoding speed in Words Per Second (WPS) ${ }^{8}$ when translating the concatenated $\rightarrow \mathrm{EN}$ valid sets on a V100 with batch size 64 and beam size 5 (averages over 3 runs). Additional speed benchmarks with other decoding settings and time spent in each component are given in Appendix Table 18. We also report chrF scores and results on more models in Appendix Table 21.

\subsection{Position of the language code}

The prevalent approach in multilingual NMT for choosing the target language is to prefix the source sequence with a language code (Johnson et al., 2017). However, it is also possible, like Tang et al. (2020), to put this code on the target side. Table 7 in Appendix analyzes the impact of the position of this language code on BLEU performance. Like observed by Wu et al. (2021), decoder-side language codes result in very low zero-shot performance in the English-centric setting. They also degrade the performance of the Base 12-2 models in all translation directions. For this reason, all our experiments use source-side language codes.

\subsection{BLEU results}

Table 1 evaluates the techniques we proposed in Section 3 on TED Talks. First, we see that the Base $12-2$ models $(3, \mathbf{6})$ perform as well or better as the Base 6-6 models $(\mathbf{2}, \mathbf{5})$ in all language directions, with a $1.7 \times$ speed boost. Multi-parallel fine-tuning $(5,6)$ significantly increases translation quality between non-English languages and incurs no drop in performance in the $\leftrightarrow \mathrm{EN}$ directions. Test-time filtering of the vocabulary with $K_{\text {test }}=10$ (see

\footnotetext{
${ }^{7}$ SacreBLEU signature:

BLEU+C.mixed+\# . 1+s. exp+tok . none+v.1.5.1

${ }^{8}$ Not BPE tokens per second, as we do not want the speed measurement to depend on the BPE tokenization used.
} 
Section 3.3) does not degrade BLEU but increases decoding speed by $30 \%$ (7). More aggressive filtering with $N_{\text {test }}=4 \mathrm{k}$ results in a drop in BLEU (8). ${ }^{9}$ The latter leads to slightly longer outputs (in terms of BPE units), which explains why it is not faster. When training with $N_{\text {train }}=4 \mathrm{k}$, we can get the same speed boost $(\mathbf{9}, \mathbf{1 0})$, without any drop in BLEU compared to models without BPE filtering $(5,6)$.

Then, we observe that Hybrid models $(\mathbf{1 1}, \mathbf{1 2})$ are slightly worse than Transformers in terms of BLEU, but are also much faster at decoding. Hybrid 12-2 (11) is $3 \times$ faster than Base 6-6 (with -0.2 BLEU on average) and $1.7 \times$ faster than Base 122 (with -0.3 BLEU). Hybrid 12-3 (12) is slower than Hybrid 12-2 and not clearly better in terms of BLEU (+0.1 BLEU).

Finally, we see that fine-tuning the Englishcentric 12-2 model into 20 language-specific shallow decoders with the multi-parallel data (14) results in the highest BLEU scores overall, with the same speed benefits as with a single shallow decoder (10). A Base 6-6 model can also be finetuned into multiple 2-layer language-specific decoders (13) and get the same performance as the single Base $6-6$ or Base 12-2 models $(\mathbf{9}, \mathbf{1 0})$. This is convenient if one wants to quickly improve the decoding speed of existing 6-6 models.

Lastly, we do a similar set of experiments within a different framework and observe the same trends (see Table 20 in Appendix).

\section{ParaCrawl Experiments}

\subsection{Data and hyper-parameters}

We scale our experiments to a more realistic setting, with the same number of languages as before, but larger amounts of training data and larger models.

We download ParaCrawl v7.1 (Bañón et al., 2020) in the 19 highest-resource languages paired with English. ${ }^{10}$ Then, like Freitag and Firat (2020), we build a multi-parallel corpus by aligning all pairs of languages through their English side. See Table 10 in Appendix for training data statistics. We train a shared BPE model with $64 \mathrm{k}$ merge operations and inline casing by sampling from this data with temperature 5 (final vocabulary size: $69 \mathrm{k}$ ).

We train the English-centric models for 1M steps

\footnotetext{
${ }^{9}$ Note that when $N=4 \mathrm{k}$, we also apply a frequency threshold of $K=10$ on BPE tokens and characters.

${ }^{10}\{\mathrm{fr}, \mathrm{de}, \mathrm{es}, \mathrm{it}, \mathrm{pt}, \mathrm{nl}, \mathrm{nb}, \mathrm{cs}, \mathrm{pl}, \mathrm{sv}, \mathrm{da}, \mathrm{el}, \mathrm{fi}, \mathrm{hr}, \mathrm{hu}, \mathrm{bg}, \mathrm{ro}$, sk, lt $\}$
}

\begin{tabular}{|c|c|c|c|c|c|}
\hline & Model & $\rightarrow \mathrm{EN}$ & $\leftarrow \mathrm{EN}$ & / EN & WPS \\
\hline \multicolumn{6}{|c|}{ SOTA (Philip et al., 2020) } \\
\hline & Bilingual & 32.4 & 24.4 & 15.0 & - \\
\hline & Best multi & 32.3 & 24.1 & 15.8 & - \\
\hline \multicolumn{6}{|c|}{ English-centric } \\
\hline 1 & Small 6-6 & 31.6 & 23.1 & 11.6 & 703 \\
\hline 2 & Base 6-6 & 31.8 & 24.2 & 13.5 & 753 \\
\hline 3 & Base 12-2 & 33.6 & 24.3 & 14.1 & 1287 \\
\hline 4 & + EN pivot & - & - & 15.4 & - \\
\hline \multicolumn{6}{|c|}{ + Multi-parallel } \\
\hline 5 & Base 6-6 & 32.8 & 24.3 & 16.3 & 732 \\
\hline 6 & Base 12-2 & 33.5 & 24.5 & 16.3 & 1203 \\
\hline 7 & $+K_{\text {test }}=10$ & 33.4 & 24.3 & 16.2 & 1539 \\
\hline 8 & $+N_{\text {test }}=4 \mathrm{k}$ & 31.5 & 22.5 & 15.2 & 1457 \\
\hline \multicolumn{6}{|c|}{+ BPE filtering $\left(N_{\text {train }}=4 \mathrm{k}\right)$} \\
\hline 9 & Base 6-6 & 32.9 & 24.2 & 16.3 & 789 \\
\hline 10 & Base 12-2 & 33.3 & 24.3 & 16.3 & 1552 \\
\hline 11 & Hybrid 12-2 & 32.8 & 23.5 & 16.1 & 2422 \\
\hline 12 & Hybrid 12-3 & 32.9 & 23.7 & 16.1 & 2145 \\
\hline \multicolumn{6}{|c|}{ + Multi-decoder } \\
\hline 13 & $6-6 \rightarrow 6-2$ & 33.0 & 24.2 & 16.0 & 1608 \\
\hline 14 & $12-2 \rightarrow 12-2$ & 33.8 & 25.1 & 16.7 & 1614 \\
\hline
\end{tabular}

Table 1: Test BLEU scores and decoding speed of TED Talks models of various depths. SOTA's "best multi" is a Transformer Small 6-6 multi-parallel model with adapter layers. WPS: speed in words per second for $\rightarrow$ EN translation. Table 21 in Appendix reports scores by more models and with additional metrics.

and fine-tune them with multi-parallel data for $200 \mathrm{k}$ more steps. Hybrid and Multi-decoder models are also fine-tuned for 200k steps from the Englishcentric models with multi-parallel data. Big 6-6 bilingual baselines are trained with the same hyperparameters for 120k steps, with joint BPE vocabularies of size 16k. More hyper-parameters are given in Appendix A.2.

The Big 6-6 and Big 12-2 English-centric models took each around 17 days to train on 4 A100s. The multi-parallel fine-tuning stages (single/multidecoder and hybrid) took $\approx 4.5$ days on $2 \mathrm{~A} 100 \mathrm{~s}$ each.

\subsection{Evaluation settings}

The ParaCrawl models are evaluated on our own valid and test splits from TED2020 (Reimers and Gurevych, 2020). ${ }^{11}$ We shuffle the parallel corpus for each translation direction and take 3000 line

\footnotetext{
${ }^{11}$ TED2020 is a different crawl from TED than that of the "TED Talks" corpus. It is more recent, it has more data and languages and it is not word-tokenized.
} 


\begin{tabular}{lc|c|c|c|c} 
& Model & $\rightarrow$ EN & $\leftarrow$ EN & $/$ EN & WPS \\
\cline { 2 - 6 } & \multicolumn{5}{c}{ English-centric } \\
\cline { 2 - 6 } $\mathbf{1 5}$ & Base 6-6 & 31.4 & 26.0 & 13.2 & 656 \\
$\mathbf{1 6}$ & Big 6-6 & 35.0 & 29.0 & 14.4 & 623 \\
$\mathbf{1 7}$ & Big 12-2 & $\mathbf{3 5 . 5}$ & $\mathbf{2 9 . 6}$ & 16.7 & 1033 \\
$\mathbf{1 8}$ & + EN pivot & - & - & 21.5 & - \\
\cline { 2 - 5 } & \multicolumn{5}{c}{ + Multi-parallel } \\
\cline { 2 - 5 } $\mathbf{1 9}$ & Big 6-6 & 34.2 & 28.3 & 20.7 & 595 \\
$\mathbf{2 0}$ & Big 12-2 & 34.9 & 29.2 & 21.3 & 1030 \\
$\mathbf{2 1}$ & $+N_{\text {test }}=16 \mathrm{k}$ & 34.8 & 29.0 & 21.2 & 1328 \\
$\mathbf{2 2}$ & $+N_{\text {test }}=8 \mathrm{k}$ & 32.9 & 27.8 & 20.4 & 1283 \\
\cline { 2 - 5 }+ BPE filtering $\left(N_{\text {train }}=8 \mathrm{k}\right)$ \\
$\mathbf{2 3}$ & Big 6-6 & 34.0 & 28.4 & 20.7 & 679 \\
$\mathbf{2 4}$ & Big 12-2 & 34.8 & 29.1 & 21.2 & 1261 \\
$\mathbf{2 5}$ & Hybrid 12-2 & 33.9 & 28.3 & 20.7 & $\mathbf{1 7 9 6}$ \\
$\mathbf{2 6}$ & Hybrid 12-3 & 34.0 & 28.3 & 20.8 & 1659 \\
\cline { 2 - 5 } & \multicolumn{5}{c}{ Multi-decoder } \\
$\mathbf{2 7}$ & $6-6 \rightarrow 6-2$ & 34.0 & 28.8 & 21.0 & 1333 \\
$\mathbf{2 8}$ & $12-2 \rightarrow 12-2$ & 35.3 & 29.4 & $\mathbf{2 1 . 8}$ & 1270
\end{tabular}

Table 2: TED2020 test BLEU scores and decoding speed of ParaCrawl models of various depths. WPS: speed in words per second for $\rightarrow \mathrm{EN}$ translation. Table 22 in Appendix reports scores by more models and with additional metrics.

pairs for the validation set and 3000 for the test set. ${ }^{12}$ To compare against the state of the art, we also provide scores on standard test sets from WMT for some language pairs. In both cases, we use SacreBLEU with its default options. ${ }^{13}$

Like in Section 4, we compute average $\rightarrow \mathrm{EN}$, $\leftarrow$ EN and / EN test BLEU scores and WPS on $\rightarrow$ EN TED2020 valid. Table 22 in Appendix reports TED2020 test chrF (Popović, 2015), as well as spBLEU scores on FLORES devtest (Goyal et al., 2021).

\subsection{BLEU results}

Table 2 shows that, like in the TED Talks experiments, the 12-2 architecture $(\mathbf{1 7}, \mathbf{2 0})$ gets as good or better BLEU scores than the standard 6-6 Transformer $(\mathbf{1 6}, \mathbf{1 9})$ and is $70 \%$ faster. It outperforms the Big 6-6 baseline in all 38 English-centric directions, both according to BLEU and chrF. Test-time BPE filtering with $N_{\text {test }}=16 \mathrm{k}$ (see Section 3.3) does not degrade BLEU (21) and improves decod-

\footnotetext{
${ }^{12}$ These splits are available for download on: https: / / europe.naverlabs.com/

research/natural-language-processing/ efficient-multilingual-machine-translation

${ }^{13}$ SacreBLEU signature:

BLEU+c.mixed+\#.1+s. exp+tok.13a+v.1.5.1
}

\begin{tabular}{c|c|c|c} 
Model & DE-EN & EN-DE & DE-FR \\
\hline Shi et al. (2020) & 40.7 & $\mathbf{4 2 . 6}$ & $\mathbf{3 5 . 4}$ \\
\hline Bilingual Big 6-6 & $\mathbf{4 2 . 3}$ & 42.0 & 34.9 \\
Mul. Big 6-6 (23) & 38.1 & 36.7 & 32.4 \\
Mul. Big 12-2 (24) & 38.4 & 37.2 & 32.6
\end{tabular}

Table 3: Comparison of our multi-parallel $\left(N_{\text {train }}=\right.$ 8k) ParaCrawl models with bilingual baselines and with the state of the art on newstest2019. Shi et al. (2020) is the top-ranking submission at WMT20 in those languages. We report their baseline scores (i.e., without back-translation, ensembling, etc.) Pivot translation with bilingual models (resp. with Big 12-2 English-centric) gives 34.2 BLEU (resp. 32.0 BLEU) on DE-FR.

ing speed by $30 \%$. However, $N_{\text {test }}=8 \mathrm{k}$ leads to a large drop in BLEU (22) without any adddional speed benefit. ${ }^{14}$ Indeed, in this setting $1.5 \%$ of the tokens that would have been generated by the nonfiltered model become out-of-vocabulary. ${ }^{15}$ This means that the filtered model has to settle for tokens that are possibly further from the true data distribution, accentuating the exposure bias (and possibly leading to degenerate outputs). Like with TED Talks, this issue is solved when training with BPE filtering (24). $N_{\text {train }}=8 \mathrm{k}$ leads to vocabularies of size 8405 on average at the cost of $4.2 \%$ longer target sequences. ${ }^{16}$ The multi-parallel Big 12-2 model with train-time BPE filtering (24) also performs better than its Big 6-6 counterpart (24) and is almost twice faster. It outperforms the latter in 370 out of 380 translation directions according to BLEU, and in 377 directions according to chrF. It also gets the same $\leftrightarrow \mathrm{EN}$ performance as the English-centric Big 6-6 model (16). Interestingly, pivot translation with an English-centric model is a strong baseline on / EN (18), slightly better than direct translation with the models fine-tuned on multi-parallel data (but also twice slower). Like on TED Talks, the Hybrid 12-2 model (25) provides a very good BLEU/speed tradeoff, matching the quality of a similar Transformer Big 6-6 model (23) at $2.6 \times$ the speed. The Big 12-2 multi-decoder model (28) slightly outperforms the single-decoder model in all directions (24), matching the $\leftrightarrow \mathrm{EN}$ performance of the best English-centric model.

\footnotetext{
${ }^{14}$ Note that when $N_{\text {train }}$ or $N_{\text {test }}$ is set, we additionally apply a frequency threshold of $K=100$ on BPE tokens and characters.

${ }^{15}$ Average number on the $\leftarrow$ EN TED2020 valid outputs of the Big 12-2 multi-parallel model.

${ }^{16}$ Average number on the training data.
} 


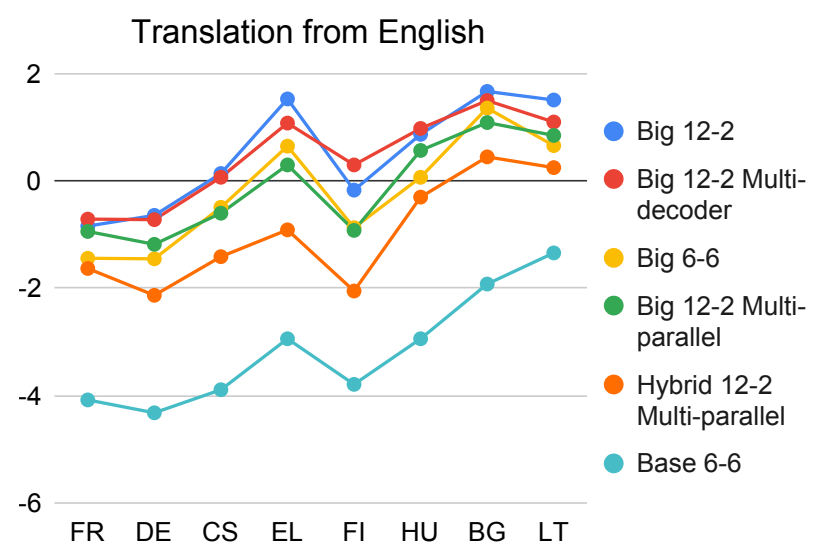

Figure 2: BLEU deltas on $\leftarrow$ EN TED2020 test (in a subset of 8 languages) by our multilingual ParaCrawl models $(17,28,16,24,25,15)$ compared to bilingual Big 6-6 baselines. The languages are sorted from highest-resource to lowest-resource.

Table 3 compares our multi-parallel models with bilingual Big 6-6 baselines and with reported numbers in the literature. It shows that bilingual models trained on ParaCrawl-only can reach similar performance as well-trained WMT baselines.

Figure 2 shows the $\leftarrow$ EN BLEU difference between our multilingual models and the ParaCrawl bilingual baselines on a subset of 8 languages. We see the same trend as in the literature: multilingual training hurts performance on high-resource languages and helps on lower-resource languages. We also see that Transformer Big 12-2 consistently outperforms Big 6-6 and that multi-parallel training consistently hurts $\leftarrow$ EN performance. Figure 6 in Appendix shows similar scores for the $\rightarrow \mathrm{EN}$ and / EN directions

\subsection{Incremental training}

Table 4 evaluates the ability of our models to be incrementally trained with a new source or target language. We see that both the single-shallowdecoder and multi-decoder models can be incrementally trained on source or target languages to reach the same or better performance as bilingual baselines. The models are incrementally trained with English-centric data only (e.g., $\mathrm{LV} \rightarrow \mathrm{EN}$ data for adding the LV source language) and yet manage to generalize to other directions ("/ EN" scores) and match the pivot-translation baseline. We can also combine new $\mathrm{X}$ source embeddings with new $\mathrm{Y}$ decoder (trained separately) to translate from $\mathrm{X}$ to $\mathrm{Y}$ and beat the pivot baseline. Note that both Latvian and Russian are close to languages known to the initial model (resp. Lithuanian and Bulgarian),

\begin{tabular}{|c|c|c|}
\hline Model & $\underset{\rightarrow \mathrm{EN}}{\mathrm{LV}}$ & $\underset{\rightarrow / \mathrm{EN}}{\mathrm{LV}}$ \\
\hline Baseline & $20.1 \quad 19.6$ & $\begin{array}{ll}16.8 & 16.1\end{array}$ \\
\hline Multi-par. (24) & 22.5 & 18.0 \\
\hline Multi-dec. (28) & 21.9 & 17.8 \\
\hline
\end{tabular}

\begin{tabular}{c|cc|cc}
\multirow{2}{*}{ Model } & \multicolumn{2}{|c|}{$\mathrm{EN} \rightarrow$} & \multicolumn{2}{c}{$/ \mathrm{EN} \rightarrow$} \\
& LV & RU & LV & RU \\
\hline Baseline & 26.6 & 23.7 & 18.5 & 16.3 \\
Multi-par. (24) & 28.1 & 23.6 & 18.6 & 16.3 \\
Multi-dec. (28) & $\mathbf{2 8 . 6}$ & $\mathbf{2 4 . 2}$ & $\mathbf{1 9 . 4}$ & $\mathbf{1 6 . 9}$
\end{tabular}

\begin{tabular}{c|cc} 
Model & $\mathrm{LV} \rightarrow \mathrm{RU}$ & $\mathrm{RU} \rightarrow \mathrm{LV}$ \\
\hline Baseline & 15.5 & 14.2 \\
Multi-par. (24) & $\mathbf{1 6 . 9}$ & $\mathbf{1 5 . 9}$ \\
Multi-dec. (28) & 16.8 & 15.7
\end{tabular}

Table 4: TED2020 test BLEU scores when incrementally adding new languages to ParaCrawl models. Top: new source language (Latvian or Russian) by training a new source embedding matrix. Middle: new target language by training a new shallow decoder. Bottom: both new source and target languages by test-time combination of source and target incrementally-trained parameters. Baseline: bilingual Big 6-6 models ("EN" columns), or pivot translation with bilingual and multilingual models.

which may help with incremental training. Similar experiments by Berard (2021) on Chinese and Arabic (not close to any known language) led to worse results than the baseline in the $\rightarrow \mathrm{EN}$ direction.

\subsection{Impact of framework}

Recent work by Narang et al. (2021) suggest that the implementation framework can change the conclusions one makes about Transformer-based architectures. In addition to a PyTorch-based framework (fairseq, Ott et al., 2019), we conduct TED Talks experiments with an in-house TensorFlow implementation, whose results are shown in Appendix (Table 20). Although BLEU and WPS values are a bit different, we observe the same trends. This confirms that our TED Talks experiments can be reproduced in a completely different framework with the same observations.

\subsection{Impact of sequence length}

When reducing the depth of the decoder, one could expect that it would have trouble generating long sequences. Figure 3 reports BLEU scores for different length buckets. We observe no abnormal patterns in any of the proposed architectures. We 


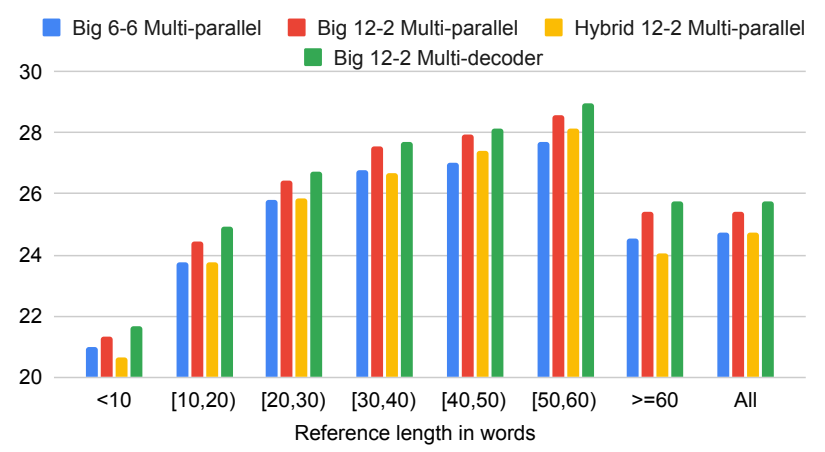

Figure 3: BLEU scores on $\leftarrow$ EN TED2020 test by the ParaCrawl models $(\mathbf{2 3}, \mathbf{2 4}, \mathbf{2 5}, \mathbf{2 8})$ according to sentence length.

first note that Big 12-2 (24) performs consistently better than Big 6-6 (23) across all sentence lengths. The performance of the Hybrid 12-2 model (25) is also consistent (slightly lower than Transformers). Figure 7 in Appendix shows scores by length in the $\rightarrow$ EN direction and with greedy decoding.

\subsection{Robustness analysis}

Even if different decoder architectures reach similar BLEU performance, some architectures might be more brittle to noise than others. To test each model's robustness, we introduce synthetic noise by either adding an unknown character (unk) randomly at the beginning, middle, or end of the sentence; or by applying 3 random char-level operations (del, ins, swap, or sub) (char). Table 5 reports the BLEU consistency ("Cy BLEU") as introduced by Niu et al. (2020) on $\leftarrow$ EN translation. ${ }^{17}$ As previously, deep-encoder / shallow decoder models (Big 12-2, Big 12-2 Multi-decoder) outperform the other architectures. BPE filtering slightly hurts robustness, despite showing close BLEU scores on the clean test sets. Additional results are given in the Appendix (Table 11).

\subsection{Human evaluation}

We conduct a human evaluation to compare the English-centric Big 6-6 and Big 12-2 models. It is done by certified professionals who are proficient in both the source and target language. We use bilingual direct assessment (DA), where raters have to evaluate the adequacy and fluency of each translation on a $0-5$ scale given the source sentence. We select a random subset of 200 sentences from newstest 2019 for DE-EN / EN-DE and from new-

\footnotetext{
${ }^{17}$ BLEU consistency measures the similarity between the translations by the same model of the clean sentence and its noised version.
}

\begin{tabular}{ll|c|c} 
Model & $\begin{array}{c}\text { Cy BLEU } \\
\text { unk }\end{array}$ & $\begin{array}{c}\text { Cy BLEU } \\
\text { char }\end{array}$ \\
\hline 19 & Big 6-6 & 73.3 & 54.2 \\
20 & Big 12-2 & $\mathbf{7 6 . 4}$ & $\mathbf{5 6 . 1}$ \\
21 & Big 12-2 $\left(N_{\text {test }}=16 \mathrm{k}\right)$ & 75.9 & 56.0 \\
23 & Big 6-6 $\left(N_{\text {train }}=8 \mathrm{k}\right)$ & 74.4 & 54.5 \\
$\mathbf{2 4}$ & Big 12-2 $\left(N_{\text {train }}=8 \mathrm{k}\right)$ & 73.7 & 55.5 \\
$\mathbf{2 5}$ & Hybrid 12-2 & 75.0 & 55.3 \\
$\mathbf{2 8}$ & Big 12-2 Multi-decoder & 76.1 & 55.4
\end{tabular}

Table 5: Robustness evaluation with average BLEU consistency (Niu et al., 2020) on $\leftarrow$ EN test sets by the multi-parallel ParaCrawl models.

\begin{tabular}{c|c|c|c} 
Model & $12-2>6-6$ & $12-2=6-6$ & $12-2<6-6$ \\
\hline EN $\rightarrow$ FR & $\mathbf{2 6 \%}$ & $51 \%$ & $23 \%$ \\
FR $\rightarrow$ EN & $\mathbf{2 5 \%}$ & $51 \%$ & $24 \%$ \\
EN $\rightarrow$ DE & $\mathbf{3 1 \%}$ & $44 \%$ & $25 \%$ \\
DE $\rightarrow$ EN & $24 \%$ & $50 \%$ & $\mathbf{2 6 \%}$
\end{tabular}

Table 6: Human evaluation on English-centric ParaCrawl models (16 and 17).

stest2014 for FR-EN / EN-FR. ${ }^{18}$ For each translation direction, 3 raters are shown all the source sentences and their translations by both systems in random order. Table 6 reports relative results averaged across the 3 raters. Big 12-2 outperforms Big 6-6 in 3 out of 4 language directions. Contrary to Kong et al. (2021) and according to both human evaluation and automatic metrics, our singleshallow-decoder model performs at least as well as the baseline model. ${ }^{19}$

\section{Conclusion}

On one hand, multilingual NMT saves training and deployment costs. On the other hand, larger architectures (required to keep performance on a par with bilingual MT) and large shared vocabularies penalize inference speed and user latency. In this work, we study various approaches to improve the speed of multilingual models without degrading translation quality. We find that Transformers with a deep encoder and a shallow decoder can outperform a baseline Transformer at a much faster decoding speed. This can be combined with per-language vocabulary filtering to reach a global $2 \times$ speed-up

\footnotetext{
${ }^{18}$ We ensure that the source sentences are original text in the corresponding language to avoid biased evaluation results due to Translationese.

${ }^{19}$ Note that our human evaluation results are only on highresource languages, but Kong et al. (2021) observed the largest BLEU drop on high-resource languages.
} 
with no loss in BLEU. A careful analysis of the results on different aspects such as sequence length, robustness to noise, and human evaluation validates this finding. Additionally, language-specific shallow decoders can be trained to get even better performance at the same speed. And finally, hybrid models with a shallow RNN decoder offer an excellent BLEU-speed trade-off $(3 \times$ faster than baseline with a minor drop in BLEU). We also provide supplementary material to facilitate reproducibility. ${ }^{20}$

\section{References}

Roee Aharoni, Melvin Johnson, and Orhan Firat. 2019. Massively multilingual neural machine translation. In Proceedings of the 2019 Conference of the North American Chapter of the Association for Computational Linguistics: Human Language Technologies, Volume 1 (Long and Short Papers), pages 3874-3884, Minneapolis, Minnesota. Association for Computational Linguistics.

Naveen Arivazhagan, Ankur Bapna, Orhan Firat, Dmitry Lepikhin, Melvin Johnson, Maxim Krikun, Mia $\mathrm{Xu}$ Chen, Yuan Cao, George Foster, Colin Cherry, et al. 2019. Massively multilingual neural machine translation in the wild: Findings and challenges. arXiv preprint arXiv:1907.05019.

Dzmitry Bahdanau, Kyunghyun Cho, and Yoshua Bengio. 2015. Neural machine translation by jointly learning to align and translate. In International Conference on Learning Representations.

Marta Bañón, Pinzhen Chen, Barry Haddow, Kenneth Heafield, Hieu Hoang, Miquel Esplà-Gomis, Mikel L. Forcada, Amir Kamran, Faheem Kirefu, Philipp Koehn, Sergio Ortiz Rojas, Leopoldo Pla Sempere, Gema Ramírez-Sánchez, Elsa Sarrías, Marek Strelec, Brian Thompson, William Waites, Dion Wiggins, and Jaume Zaragoza. 2020. ParaCrawl: Web-scale acquisition of parallel corpora. In Proceedings of the 58th Annual Meeting of the Association for Computational Linguistics, pages 4555-4567, Online. Association for Computational Linguistics.

Ankur Bapna and Orhan Firat. 2019. Simple, scalable adaptation for neural machine translation. In Proceedings of the 2019 Conference on Empirical Methods in Natural Language Processing and the 9th International Joint Conference on Natural Language Processing (EMNLP-IJCNLP), pages 15381548 , Hong Kong, China. Association for Computational Linguistics.

Maximiliana Behnke and Kenneth Heafield. 2020. Losing heads in the lottery: Pruning transformer attention in neural machine translation. In Proceedings of

\footnotetext{
${ }^{20}$ https: / / europe. naverlabs.com/

research/natural-language-processing/

efficient-multilingual-machine-translation
}

the 2020 Conference on Empirical Methods in Natural Language Processing (EMNLP), pages 26642674, Online. Association for Computational Linguistics.

Alexandre Berard. 2021. Continual learning in multilingual NMT via language-specific embeddings. In Proceedings of the Sixth Conference on Machine Translation, Online. Association for Computational Linguistics.

Alexandre Berard, Ioan Calapodescu, and Claude Roux. 2019. Naver Labs Europe's systems for the WMT19 machine translation robustness task. In Proceedings of the Fourth Conference on Machine Translation (Volume 2: Shared Task Papers, Day 1), pages 526-532, Florence, Italy. Association for Computational Linguistics.

Isaac Caswell and Google Research Bowen Liang, Software Engineers. 2020. Recent advances in Google Translate.

Mia Xu Chen, Orhan Firat, Ankur Bapna, Melvin Johnson, Wolfgang Macherey, George Foster, Llion Jones, Mike Schuster, Noam Shazeer, Niki Parmar, Ashish Vaswani, Jakob Uszkoreit, Lukasz Kaiser, Zhifeng Chen, Yonghui Wu, and Macduff Hughes. 2018. The best of both worlds: Combining recent advances in neural machine translation. In Proceedings of the 56th Annual Meeting of the Association for Computational Linguistics (Volume 1: Long Papers), pages 76-86, Melbourne, Australia. Association for Computational Linguistics.

Shuoyang Ding, Adithya Renduchintala, and Kevin Duh. 2019. A call for prudent choice of subword merge operations in neural machine translation. In Proceedings of Machine Translation Summit XVII Volume 1: Research Track, pages 204-213, Dublin, Ireland. European Association for Machine Translation.

Carlos Escolano, Marta R. Costa-jussà, José A. R. Fonollosa, and Mikel Artetxe. 2021. Multilingual machine translation: Closing the gap between shared and language-specific encoder-decoders. In Proceedings of the 16th Conference of the European Chapter of the Association for Computational Linguistics: Main Volume, pages 944-948, Online. Association for Computational Linguistics.

Angela Fan, Shruti Bhosale, Holger Schwenk, Zhiyi Ma, Ahmed El-Kishky, Siddharth Goyal, Mandeep Baines, Onur Celebi, Guillaume Wenzek, Vishrav Chaudhary, et al. 2020. Beyond English-centric multilingual machine translation. arXiv preprint arXiv:2010.11125.

Markus Freitag and Orhan Firat. 2020. Complete multilingual neural machine translation. In Proceedings of the Fifth Conference on Machine Translation, pages 550-560, Online. Association for Computational Linguistics. 
Naman Goyal, Cynthia Gao, Vishrav Chaudhary, PengJen Chen, Guillaume Wenzek, Da Ju, Sanjana Krishnan, Marc'Aurelio Ranzato, Francisco Guzman, and Angela Fan. 2021. The FLORES-101 evaluation benchmark for low-resource and multilingual machine translation. arXiv preprint arXiv:2106.03193.

Yi-Te Hsu, Sarthak Garg, Yi-Hsiu Liao, and Ilya Chatsviorkin. 2020. Efficient inference for neural machine translation. In Proceedings of SustaiNLP: Workshop on Simple and Efficient Natural Language Processing, pages 48-53, Online. Association for Computational Linguistics.

Melvin Johnson, Mike Schuster, Quoc V. Le, Maxim Krikun, Yonghui Wu, Zhifeng Chen, Nikhil Thorat, Fernanda Viégas, Martin Wattenberg, Greg Corrado, Macduff Hughes, and Jeffrey Dean. 2017. Google's multilingual neural machine translation system: Enabling zero-shot translation. Transactions of the Association for Computational Linguistics, 5:339-351.

Jungo Kasai, Nikolaos Pappas, Hao Peng, James Cross, and Noah Smith. 2021a. Deep encoder, shallow decoder: Reevaluating non-autoregressive machine translation. In International Conference on Learning Representations.

Jungo Kasai, Hao Peng, Yizhe Zhang, Dani Yogatama, Gabriel Ilharco, Nikolaos Pappas, Yi Mao, Weizhu Chen, and Noah A Smith. 2021b. Finetuning pretrained transformers into RNNs. arXiv preprint arXiv:2103.13076.

Young Jin Kim, Marcin Junczys-Dowmunt, Hany Hassan, Alham Fikri Aji, Kenneth Heafield, Roman Grundkiewicz, and Nikolay Bogoychev. 2019. From research to production and back: Ludicrously fast neural machine translation. In Proceedings of the 3rd Workshop on Neural Generation and Translation, pages 280-288, Hong Kong. Association for Computational Linguistics.

Xiang Kong, Adithya Renduchintala, James Cross, Yuqing Tang, Jiatao Gu, and Xian Li. 2021. Multilingual neural machine translation with deep encoder and multiple shallow decoders. In Proceedings of the 16th Conference of the European Chapter of the Association for Computational Linguistics. Main Volume, pages 1613-1624, Online. Association for Computational Linguistics.

Tao Lei. 2021. When attention meets fast recurrence: Training language models with reduced compute. arXiv preprint arXiv:2102.12459.

Bei Li, Ziyang Wang, Hui Liu, Yufan Jiang, Quan Du, Tong Xiao, Huizhen Wang, and Jingbo Zhu. 2020. Shallow-to-deep training for neural machine translation. In Proceedings of the 2020 Conference on Empirical Methods in Natural Language Processing (EMNLP), pages 995-1005, Online. Association for Computational Linguistics.
Yanyang Li, Ye Lin, Tong Xiao, and Jingbo Zhu. 2021. An efficient transformer decoder with compressed sub-layers. arXiv preprint arXiv:2101.00542.

Liyuan Liu, Xiaodong Liu, Jianfeng Gao, Weizhu Chen, and Jiawei Han. 2020. Understanding the difficulty of training transformers. In Proceedings of the 2020 Conference on Empirical Methods in Natural Language Processing (EMNLP), pages 57475763, Online. Association for Computational Linguistics.

Sungwon Lyu, Bokyung Son, Kichang Yang, and Jaekyoung Bae. 2020. Revisiting Modularized Multilingual NMT to Meet Industrial Demands. In Proceedings of the 2020 Conference on Empirical Methods in Natural Language Processing (EMNLP), pages 5905-5918, Online. Association for Computational Linguistics.

Sharan Narang, Hyung Won Chung, Yi Tay, William Fedus, Thibault Fevry, Michael Matena, Karishma Malkan, Noah Fiedel, Noam Shazeer, Zhenzhong Lan, et al. 2021. Do Transformer modifications transfer across implementations and applications? arXiv preprint arXiv:2102.11972.

Xing Niu, Prashant Mathur, Georgiana Dinu, and Yaser Al-Onaizan. 2020. Evaluating Robustness to Input Perturbations for Neural Machine Translation. In Proceedings of the 58th Annual Meeting of the Association for Computational Linguistics, pages 85388544, Online. Association for Computational Linguistics.

Myle Ott, Sergey Edunov, Alexei Baevski, Angela Fan, Sam Gross, Nathan Ng, David Grangier, and Michael Auli. 2019. fairseq: A fast, extensible toolkit for sequence modeling. In Proceedings of the 2019 Conference of the North American Chapter of the Association for Computational Linguistics (Demonstrations), pages 48-53, Minneapolis, Minnesota. Association for Computational Linguistics.

Jerin Philip, Alexandre Berard, Matthias Gallé, and Laurent Besacier. 2020. Monolingual adapters for zero-shot neural machine translation. In Proceedings of the 2020 Conference on Empirical Methods in Natural Language Processing (EMNLP), pages 4465-4470, Online. Association for Computational Linguistics.

Maja Popović. 2015. chrF: character n-gram F-score for automatic MT evaluation. In Proceedings of the Tenth Workshop on Statistical Machine Translation, pages 392-395, Lisbon, Portugal. Association for Computational Linguistics.

Ye Qi, Devendra Sachan, Matthieu Felix, Sarguna Padmanabhan, and Graham Neubig. 2018. When and why are pre-trained word embeddings useful for neural machine translation? In Proceedings of the 2018 Conference of the North American Chapter of the Association for Computational Linguistics: Human Language Technologies, Volume 2 (Short Papers), 
pages 529-535, New Orleans, Louisiana. Association for Computational Linguistics.

Nils Reimers and Iryna Gurevych. 2020. Making monolingual sentence embeddings multilingual using knowledge distillation. In Proceedings of the 2020 Conference on Empirical Methods in Natural Language Processing (EMNLP), pages 4512-4525, Online. Association for Computational Linguistics.

Jean Senellart, Dakun Zhang, Bo Wang, Guillaume Klein, Jean-Pierre Ramatchandirin, Josep Crego, and Alexander Rush. 2018. OpenNMT system description for WNMT 2018: 800 words/sec on a single-core CPU. In Proceedings of the $2 n d$ Workshop on Neural Machine Translation and Generation, pages 122-128, Melbourne, Australia. Association for Computational Linguistics.

Tingxun Shi, Shiyu Zhao, Xiaopu Li, Xiaoxue Wang, Qian Zhang, Di Ai, Dawei Dang, Xue Zhengshan, and Jie Hao. 2020. OPPO's machine translation systems for WMT20. In Proceedings of the Fifth Conference on Machine Translation, pages 282-292, Online. Association for Computational Linguistics.

Xing Shi and Kevin Knight. 2017. Speeding up neural machine translation decoding by shrinking runtime vocabulary. In Proceedings of the 55th Annual Meeting of the Association for Computational Linguistics (Volume 2: Short Papers), pages 574-579, Vancouver, Canada. Association for Computational Linguistics.

Raphael Shu and Hideki Nakayama. 2017. Compressing word embeddings via deep compositional code learning. arXiv preprint arXiv:1711.01068.

Yuqing Tang, Chau Tran, Xian Li, Peng-Jen Chen, Naman Goyal, Vishrav Chaudhary, Jiatao Gu, and Angela Fan. 2020. Multilingual translation with extensible multilingual pretraining and finetuning. arXiv preprint arXiv:2008.00401.

Liwei $\mathrm{Wu}$, Shanbo Cheng, Mingxuan Wang, and Lei Li. 2021. Language tags matter for zero-shot neural machine translation. In Findings of the Association for Computational Linguistics: ACL-IJCNLP 2021, pages 3001-3007, Online. Association for Computational Linguistics.

Biao Zhang, Ankur Bapna, Rico Sennrich, and Orhan Firat. 2021. Share or not? learning to schedule language-specific capacity for multilingual translation. In International Conference on Learning Representations.

Biao Zhang, Philip Williams, Ivan Titov, and Rico Sennrich. 2020. Improving massively multilingual neural machine translation and zero-shot translation. In Proceedings of the 58th Annual Meeting of the Association for Computational Linguistics, pages 16281639, Online. Association for Computational Linguistics. 


\section{A Appendix}

\section{A.1 Position of the language code}

Table 7 analyzes the impact of the language code position on BLEU performance. With the Base 6-6 architecture, decoder-side codes perform approximately as well as encoder-side codes (except for zeroshot translation). However, with the Base 12-2 architecture, decoder-side codes result in a noticeable drop in performance in most directions. Indeed, when the lang code is on the source side, the deep encoder knows the target language and can start "translating." When it is on the target side, the encoder has no way of knowing which language to start translating into. So it outputs a universal representation that is harder to transform into a target-language sentence by the limited-capacity shallow decoder. Note that $\rightarrow$ EN performance in the English-centric setting is not affected. We believe this is because the encoder can easily guess that the target language is English by detecting the language of the input. We believe this is also the reason for the low zero-shot performance: the encoder starts translating all non-English inputs into English, and the decoder receives a representation that it cannot translate into other languages than English.

\begin{tabular}{cl|c|c|c|c} 
& Lang code position & Model & $\rightarrow$ EN & $\leftarrow$ EN & $/$ EN \\
\hline \multicolumn{6}{c}{ English-centric } \\
\hline $\mathbf{2}$ & Encoder & Base 6-6 & 31.8 & 24.2 & 13.5 \\
$\mathbf{2 9}$ & Decoder & Base 6-6 & 32.0 & 23.9 & 5.43 \\
$\mathbf{3}$ & Encoder & Base 12-2 & $\mathbf{3 3 . 6}$ & $\mathbf{2 4 . 3}$ & $\mathbf{1 4 . 1}$ \\
$\mathbf{3 0}$ & Decoder & Base 12-2 & 33.5 & 22.8 & 0.74 \\
\hline \multicolumn{6}{c}{ Multi-parallel + BPE filtering $\left(N_{\text {train }}=4 \mathrm{k}\right)$} \\
\hline $\mathbf{9}$ & Encoder & Base 6-6 & 32.9 & 24.2 & 16.3 \\
$\mathbf{3 1}$ & Decoder & Base 6-6 & 32.7 & 23.9 & 16.2 \\
$\mathbf{1 0}$ & Encoder & Base 12-2 & $\mathbf{3 3 . 3}$ & $\mathbf{2 4 . 3}$ & $\mathbf{1 6 . 3}$ \\
$\mathbf{3 2}$ & Decoder & Base 12-2 & 32.5 & 23.0 & 15.7
\end{tabular}

Table 7: Test BLEU scores of TED Talks models with encoder-side or decoder-side language codes. Like Tang et al. (2020), we implement decoder-side lang codes by replacing BOS (i.e., the first embedding input to the decoder) with the lang code.

\section{A.2 Framework and hyper-parameters}

We do our experiments in the fairseq v0.10.2 framework (Ott et al., 2019), which we modify to implement on-the-fly pre-processing and sampling from multilingual corpora.

We randomly sample language pairs with $p_{k}=\frac{D_{k}^{1 / T}}{\sum D_{i}^{1 / T}}$ where $D_{k}$ is the number of sentence pairs for language pair $k$ and $T$ is the temperature (Arivazhagan et al., 2019). Tables 8 and 10 give the resulting sampling probabilities by target language. We build heterogeneous batches using this sampling strategy (i.e., containing any mixture of languages), by sampling 100k sentence pairs at a time and sorting them by length into batches. Language-specific decoders are trained with homogeneous batches with respect to the target language (we increase the "buffer size" to $1 \mathrm{M}$ and group sentence pairs by target language before batching).

Tables 12 and 13 give the fairseq hyperparameters of our TED Talks and ParaCrawl Transformer models. Tables 14 and 15 give the training details of the fine-tuned models.

Our Hybrid models use a variant of the hybrid RNMT+ architecture proposed by Chen et al. (2018). Contrary to them, we use single-head additive attention (Bahdanau et al., 2015); sum the attention and LSTM output before the vocabulary projection; and apply layer normalization on the input of the LSTMs (rather than on the gates). We apply the same amounts of dropout as in the Transformer but on both the LSTM outputs (except for the first LSTM) and the target embeddings. 


\begin{tabular}{c|c|c|c} 
Language & Code & English-centric lines & Multi-parallel lines \\
\hline English & en & $3,556,962$ & $3,556,962$ \\
Arabic & ar & 214,111 & $3,430,385$ \\
Hebrew & he & 211,819 & $3,399,679$ \\
Russian & ru & 208,458 & $3,379,440$ \\
Korean & ko & 205,640 & $3,350,599$ \\
Italian & it & 204,503 & $3,350,483$ \\
Japanese & ja & 204,090 & $3,312,997$ \\
Mandarin Chinese & zh_cn & 199,855 & $3,297,628$ \\
Spanish & es & 196,026 & $3,234,798$ \\
French & fr & 192,304 & $3,192,551$ \\
Brazilian Portuguese & pt_br & 184,755 & $3,110,048$ \\
Dutch & nl & 183,767 & $3,053,593$ \\
Turkish & tr & 182,470 & $3,017,706$ \\
Romanian & ro & 180,484 & $3,055,943$ \\
Polish & pl & 176,169 & $3,002,206$ \\
Bulgarian & bg & 174,444 & $2,946,693$ \\
Vietnamese & vi & 171,995 & $2,807,695$ \\
German & de & 167,888 & $2,900,115$ \\
Persian & fa & 150,965 & $2,405,646$ \\
Hungarian & hu & 147,219 & $2,465,081$ \\
\hline Total & all & $7,113,924$ & $62,270,248$
\end{tabular}

Table 8: Size of the Top 20 TED Talks corpus. English has 253,292 unique lines. The average English sentence length is 21.1 words and 26.7 wordpieces.

\begin{tabular}{|c|c|c|c|c|}
\hline & Model & $\rightarrow \mathrm{EN}$ & $\leftarrow \mathrm{EN}$ & / EN \\
\hline \multicolumn{5}{|c|}{ English-centric } \\
\hline 2 & Base $6-6$ post-norm & 32.1 & 24.5 & 13.6 \\
\hline 3 & Base $12-2$ post-norm pre-trained & 33.7 & 24.5 & 14.1 \\
\hline 33 & Base $12-2$ pre-norm & 33.5 & 24.3 & 12.6 \\
\hline 34 & Base $12-2$ enc pre-norm & 33.3 & 24.1 & 13.1 \\
\hline 35 & Base 18 -1 enc pre-norm ${ }^{\star}$ & 31.6 & 22.8 & 12.1 \\
\hline
\end{tabular}

Table 9: Valid BLEU scores of English-centric TED Talks models with deep encoders, depending on the training strategy used. $\star$ : this model was stopped before the end (after 60 epochs).
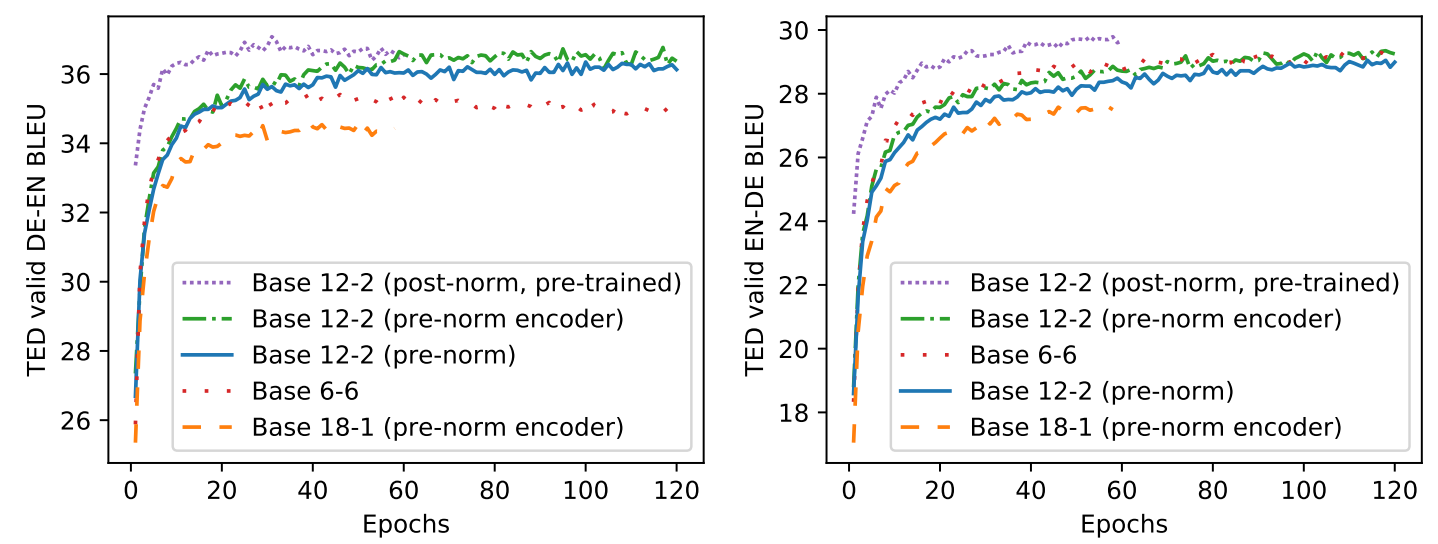

Figure 4: Training progress of English-centric models trained on TED Talks. The names in the legend are sorted from highest to lowest BLEU score. The pre-trained 12-2 model was initialized with the 6-6 model's checkpoint at epoch 60 and fine-tuned for 60 more epochs. The 18-1 model was stopped in the middle of training. 

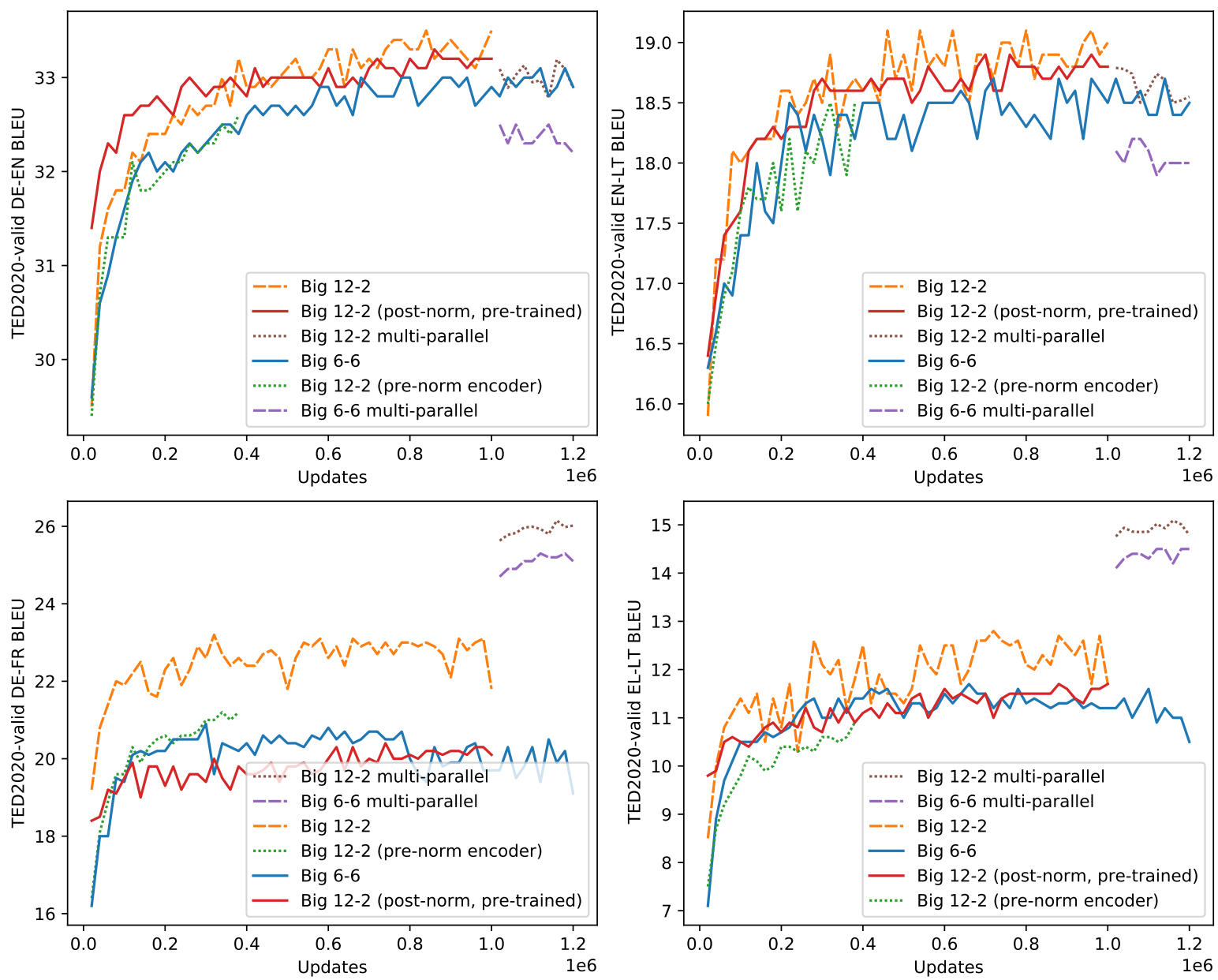

Figure 5: Training progress of English-centric and multi-parallel models trained on ParaCrawl (without BPE filtering). The names in the legend are sorted from highest to lowest BLEU score. The pre-trained 12-2 model was initialized with the 6-6 model's checkpoint at step 1M and fine-tuned for 1M more steps.
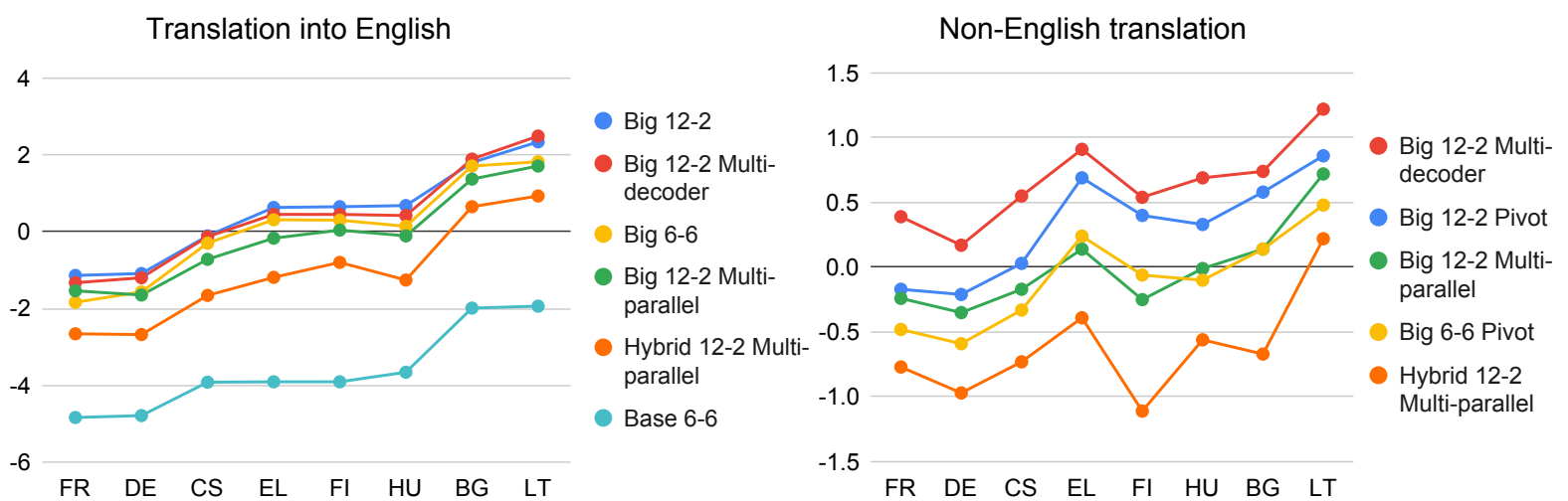

Figure 6: BLEU deltas on TED2020 test (in a subset of 8 languages) by our multilingual ParaCrawl models (17, 28, 16, 24, 25, 15) compared to pivot translation through English with bilingual Big 6-6 baselines. The "nonEnglish" score for language $\mathrm{X}$ is the average of test scores from all the other languages (except English) into X. The languages are sorted from highest-resource to lowest-resource (in terms of English-centric data amounts). 


\begin{tabular}{c|c|c|c|c|c|c} 
& \multirow{2}{*}{ Language } & Code & Family & \multicolumn{2}{|c}{ English-centric } & \multicolumn{2}{c}{ Multi-parallel } \\
& & & Lines & Trg. prob (T=5) & Lines & Trg. prob (T=2) \\
\hline English & en & Germanic & $450,298,290$ & 0.500 & $450,298,290$ & 0.109 \\
French & fr & Romance & $95,432,158$ & 0.038 & $215,631,123$ & 0.070 \\
German & de & Romance & $76,490,492$ & 0.036 & $192,673,679$ & 0.068 \\
Spanish & es & Romance & $72,973,508$ & 0.036 & $191,713,598$ & 0.068 \\
Italian & it & Romance & $38,054,969$ & 0.031 & $136,107,553$ & 0.061 \\
Portuguese & pt & Romance & $29,181,190$ & 0.030 & $117,682,221$ & 0.058 \\
Dutch & nl & Germanic & $27,361,570$ & 0.029 & $104,353,586$ & 0.055 \\
Norwegian & nb & Germanic & $15,384,700$ & 0.026 & $65,367,888$ & 0.045 \\
Czech & cs & Slavic & $12,922,615$ & 0.025 & $65,547,562$ & 0.046 \\
Polish & pl & Slavic & $12,877,872$ & 0.025 & $69,265,211$ & 0.047 \\
Swedish & sv & Germanic & $10,969,372$ & 0.025 & $60,160,949$ & 0.044 \\
Danish & da & Germanic & $9,792,687$ & 0.024 & $61,276,295$ & 0.044 \\
Greek & el & Hellenic & $8,915,258$ & 0.024 & $48,294,800$ & 0.039 \\
Finnish & fi & Uralic & $6,833,568$ & 0.022 & $47,624,701$ & 0.040 \\
Croatian & hr & Slavic & $6,338,125$ & 0.022 & $30,467,579$ & 0.031 \\
Hungarian & hu & Uralic & $6,294,289$ & 0.022 & $42,527,237$ & 0.037 \\
Bulgarian & bg & Slavic & $6,098,653$ & 0.022 & $36,835,445$ & 0.035 \\
Romanian & ro & Romance & $5,786,263$ & 0.022 & $40,521,359$ & 0.037 \\
Slovak & sk & Slavic & $4,557,803$ & 0.021 & $36,387,740$ & 0.035 \\
Lithuanian & lt & Baltic & $4,033,198$ & 0.020 & $30,205,598$ & 0.032 \\
\hline Total & all & - & $900,596,580$ & 1.0 & $2,042,942,414$ & 1.0 \\
\hline Russian ${ }^{\star}$ & ru & Slavic & $5,120,207$ & - & - & - \\
Latvian $^{n}$ & lv & Baltic & $3,607,272$ & - & - & - \\
\hline
\end{tabular}

Table 10: Size of the Top 20 ParaCrawl corpus and target language sampling probabilities in its English-centric setting and its multi-parallel setting. $\mathrm{T}=$ sampling temperature. English has 271,851,754 unique lines. $\star$ : all languages use the latin script, except for Greek (Greek alphabet) and Bulgarian/Russian (Cyrillic). The average English sentence length is 19.0 words and 30.4 wordpieces.

\begin{tabular}{|c|c|c|c|c|}
\hline Model & $\begin{array}{l}\text { BLEU } \\
\text { unk }\end{array}$ & $\begin{array}{l}\text { BLEU Consistency } \\
\text { unk }\end{array}$ & $\begin{array}{l}\text { BLEU } \\
\text { char }\end{array}$ & $\begin{array}{l}\text { BLEU Consistency } \\
\text { char }\end{array}$ \\
\hline 19 Big 6-6 & 24.2 & 73.3 & 19.5 & 54.2 \\
\hline 20 Big 12-2 & 26.0 & 76.4 & 21.1 & 56.1 \\
\hline $21 \operatorname{Big} 12-2\left(N_{\text {test }}=16 \mathrm{k}\right)$ & 26.0 & 75.9 & 21.0 & 56.0 \\
\hline 23 Big 6-6 $\left(N_{\text {train }}=8 \mathrm{k}\right)$ & 24.4 & 74.4 & 19.6 & 54.5 \\
\hline 24 Big $12-2\left(N_{\text {train }}=8 \mathrm{k}\right)$ & 25.6 & 73.7 & 20.9 & 55.5 \\
\hline 25 Hybrid $12-2\left(N_{\text {train }}=8 \mathrm{k}\right)$ & 24.8 & 75.0 & 20.4 & 55.3 \\
\hline 26 Hybrid $12-3\left(N_{\text {train }}=8 \mathrm{k}\right)$ & 25.1 & 76.5 & 20.2 & 55.0 \\
\hline
\end{tabular}

Table 11: Robustness evaluation on $\leftarrow$ EN test sets of the multi-parallel ParaCrawl models. "Unk" adds one unknown symbol at a random position in each test sentence and "char" does 3 random character-level operations per sentence. "BLEU consistency" by Niu et al. (2020) is a BLEU score between the translations of the clean and noisy versions of the same test set by a given model. 


\begin{tabular}{|cc|}
\hline Parametername & Parameter value \\
\hline share_all_embeddings & True \\
arch & transformer \\
lr_scheduler & inverse_sqrt \\
optimizer & adam \\
adam_betas & $0.9,0.999$ \\
fp16 & True \\
clip_norm & 0.0 \\
lr & $0.0005^{\star}$ \\
warmup_updates & 4000 \\
warmup_init_lr & $1 \mathrm{e}-07$ \\
criterion & label_smoothed_cross_entropy \\
label_smoothing & 0.1 \\
dropout & $0.3^{\star}$ \\
max_tokens & 4000 \\
max_epoch & $120^{\star}$ \\
save-interval & 1 \\
validate-interval & 1 \\
keep-last-epochs & 1 \\
update_freq & $4^{\dagger}$ \\
\hline lang_temperature & 5 \\
decoder_dropout & $0.3^{\star}$ \\
\hline
\end{tabular}

Table 12: fairseq hyper-parameters of the Base 6-6 TED Talks models. $\dagger$ : we normalize this value by the number of GPUs to have a constant batch size. For instance, models trained on 4 GPUs use update_freq=1. $\star$ : as shown in Table 14, the fine-tuned models use different values for these parameters. The Small 6-6 models use the transformer_iwslt_de_en architecture.

\begin{tabular}{|cc|}
\hline max_source_positions & 256 \\
max_target_positions & 256 \\
share_all_embeddings & True \\
arch & transformer_vaswani_wmt_en_de_big \\
lr_scheduler & inverse_sqrt \\
optimizer & adam \\
adam_betas & $0.9,0.98$ \\
fp16 & True \\
clip_norm & 1.0 \\
lr & $0.0005^{\star}$ \\
warmup_updates & 4000 \\
warmup_init_lr & $1 \mathrm{e}-07$ \\
criterion & 0.1 \\
label_smoothing & 0.1 \\
dropout & 8000 \\
max_tokens & $1000000^{\star}$ \\
max_update & 20000 \\
save_interval_updates & 20000 \\
validate_interval_updates & $32^{\dagger}$ \\
update_freq & $5^{\ddagger}$ \\
\hline lang_temperature & \\
\hline
\end{tabular}

Table 13: fairseq hyper-parameters of the Big 6-6 ParaCrawl models. $\dagger$ : we normalize this value by the number of GPUs to have a constant batch size. For instance, models trained on 4 GPUs use update_freq=8. $\neq$ : we use a temperature of 2 in the multi-parallel (or multi-decoder) finetuning stage. $\star$ : as shown in Table 15, the fine-tuned models use different values for these parameters. 


\begin{tabular}{l|c|c|c|c|c}
\multicolumn{1}{c|}{ Models } & Initialized with & LR reset & Dropout & Data & Epochs \\
\hline Base 6-6 (2) & - & - & 0.3 & TED-EN & 120 \\
Base 6-2 (36) & - & - & 0.3 & TED-EN & 120 \\
Base 12-2 (3) & $(\mathbf{2}) @ 60$ & yes $(0.0005)$ & 0.3 & TED-EN & 60 \\
Base 6-6 Multi-parallel (5, 9) & $\mathbf{( 2 )} @ 120$ & no & 0.1 & TED-ALL & 10 \\
Base 12-2 Multi-parallel (6, 10) & $\mathbf{( 3 )} @ 60$ & no & 0.1 & TED-ALL & 10 \\
Hybrid Multi-parallel (11, 12) & $\mathbf{( 3 )} @ 60$ & yes $(0.0003)$ & 0.1 & TED-ALL & 10 \\
Base 12-2 Multi-decoder (14) & $\mathbf{( 3 ) @ 6 0}$ & yes $(0.0001)$ & $0.1 / 0.3^{\star}$ & TED-ALL & 10
\end{tabular}

Table 14: Details about multi-stage training of TED Talks models. $\star$ : different dropout values in the encoder and decoders.

\begin{tabular}{l|c|c|c|c}
\multicolumn{1}{c|}{ Models } & Initialized with & LR reset & Data & Updates \\
\hline Big 6-6 (16) & - & - & Para-EN & $1 \mathrm{M}$ \\
Big 6-2 (37) & - & - & Para-EN & $1 \mathrm{M}$ \\
Big 12-2 (17) & - & - & Para-EN & $1 \mathrm{M}$ \\
Big 6-6 Multi-parallel (19, 23) & $(\mathbf{1 6}) @ 1 \mathrm{M}$ & no & Para-ALL & $200 \mathrm{k}$ \\
Big 12-2 Multi-parallel (20, 24) & $\mathbf{( 1 7 )} @ 1 \mathrm{M}$ & no & Para-ALL & $200 \mathrm{k}$ \\
Hybrid Multi-parallel (25, 26) & $\mathbf{( 1 7 )} @ 1 \mathrm{M}$ & yes (0.0005) & Para-ALL & 200k \\
Big 12-2 Multi-decoder (28) & $(\mathbf{1 7 )} @ 1 \mathrm{M}$ & yes (0.0005) & Para-ALL & 200k
\end{tabular}

Table 15: Details about multi-stage training of ParaCrawl models.

\begin{tabular}{l|c|c}
\multicolumn{1}{c}{ Models } & Params without embeddings $(\mathrm{M})$ & Embeddings $(\mathrm{M})$ \\
\hline Base 6-6 $(\mathbf{2}, \mathbf{5})$ & $44.1(18.9+25.2)$ & 36.0 \\
Base 6-2 $\mathbf{( 3 6}$ & $27.3(18.9+8.4)$ & 36.0 \\
Base 12-2 (3, 6) & $46.2(37.8+8.4)$ & 36.0 \\
Base 12-2 $(K=10)(\mathbf{7})$ & 46.2 & $36.0+76.2$ \\
Base 12-2 $(N=4 \mathrm{k})(\mathbf{8}, \mathbf{1 0})$ & 46.2 & $36.0+45.4$ \\
Hybrid 12-2 $(N=4 \mathrm{k})(\mathbf{1 1})$ & $43.6(37.8+5.8)$ & $36.0+45.4$ \\
Hybrid 12-3 $(N=4 \mathrm{k})(\mathbf{1 2})$ & $46.8(37.8+8.9)$ & $36.0+45.4$ \\
Multi-decoder Base 12-2 $(N=4 \mathrm{k})(\mathbf{1 4})$ & $206.0(37.8+20 \times 8.4)$ & $36.0+45.4$
\end{tabular}

Table 16: Number of parameters in the TED Talks models.

\begin{tabular}{l|c|c}
\multicolumn{1}{c}{ Models } & Params without embeddings $(\mathrm{M})$ & Embeddings $(\mathrm{M})$ \\
\hline Big 6-6 (16, 19) & $176.4(75.6+100.8)$ & 70.7 \\
Big 6-2 (37) & $109.2(75.6+33.6)$ & 70.7 \\
Big 12-2 (17, 20) & $184.7(151.1+33.6)$ & 70.7 \\
Big 12-2 $(N=16 \mathrm{k})(\mathbf{2 1})$ & 184.7 & $70.7+332.3$ \\
Big 12-2 $(N=8 \mathrm{k})(\mathbf{2 2}, \mathbf{2 4})$ & 184.7 & $70.7+172.2$ \\
Hybrid 12-2 $(N=8 \mathrm{k})(\mathbf{2 5})$ & $174.2(151.1+23.1)$ & $70.7+172.2$ \\
Hybrid 12-3 $(N=8 \mathrm{k})(\mathbf{2 6})$ & $186.8(151.1+35.7)$ & $70.7+172.2$ \\
Multi-decoder Big 12-2 $(N=8 \mathrm{k})(\mathbf{2 8})$ & $823.0(151.2+20 \times 33.6)$ & $70.7+172.2$
\end{tabular}

Table 17: Number of parameters in the ParaCrawl models. 


\begin{tabular}{|c|c|c|c|c|c|c|}
\hline \multirow[b]{2}{*}{ Time (s) } & \multirow[b]{2}{*}{ Parameters } & \multicolumn{3}{|c|}{ English-centric } & \multicolumn{2}{|c|}{ Multi-parallel $\left(N_{\text {train }}=4 \mathrm{k}\right)$} \\
\hline & & $\begin{array}{c}\text { Base 6-6 } \\
\text { (2) }\end{array}$ & $\begin{array}{c}\text { Base 6-2 } \\
-\end{array}$ & $\begin{array}{l}\text { Base 12-2 } \\
\quad(\mathbf{3})\end{array}$ & $\begin{array}{c}\text { Base } 12-2 \\
\quad(\mathbf{1 0})\end{array}$ & $\begin{array}{c}\text { Hybrid 12-2 } \\
\text { (11) }\end{array}$ \\
\hline \multirow{4}{*}{ Total } & beam $=1 \mathrm{bs}=1$ & 18259 & 8455 & 9177 & 8483 & 5227 \\
\hline & beam $=5 \mathrm{bs}=1$ & 34216 & 18215 & 18861 & 15351 & 10617 \\
\hline & beam $=1 \mathrm{bs}=64$ & 1364 & 555 & 551 & 622 & 273 \\
\hline & \multirow{7}{*}{ beam $=5 \mathrm{bs}=64$} & 2330 & 1334 & 1329 & 1050 & 691 \\
\hline Encoder & & 15 & 15 & 29 & 30 & 29 \\
\hline Decoder & & 1435 & 554 & 545 & 548 & 234 \\
\hline Self-attn / RNN & & 477 & 162 & 159 & 168 & 71 \\
\hline Cross-attn & & 399 & 133 & 132 & 139 & 60 \\
\hline Softmax & & 49 & 48 & 48 & 21 & 21 \\
\hline Beam top-k & & 350 & 341 & 334 & 38 & 38 \\
\hline
\end{tabular}

Table 18: Time benchmark across different TED Talks model sizes and decoding settings. Time in seconds spent decoding the concatenation of all $\mathrm{X} \rightarrow \mathrm{EN}$ TED valid sets (averages over 3 runs). Note that to mimic a true online setting, no sorting by length is applied (i.e., buffer_size=batch_size). We modify fairseq's code to avoid the slow beam search code when beam $=1$ (which unnecessarily computes and stores log probabilities). We see that in this setting, vocabulary size has a minor impact on speed.

\begin{tabular}{|c|c|c|c|c|c|c|}
\hline \multirow[b]{2}{*}{ Time (s) } & \multirow[b]{2}{*}{ Parameters } & \multicolumn{3}{|c|}{ English-centric } & \multicolumn{2}{|c|}{ Multi-parallel $\left(N_{\text {train }}=8 \mathrm{k}\right)$} \\
\hline & & $\begin{array}{l}\text { Big 6-6 } \\
\text { (16) }\end{array}$ & $\begin{array}{l}\text { Big 6-2 } \\
(\mathbf{3 7})\end{array}$ & $\begin{array}{l}\text { Big 12-2 } \\
\quad(\mathbf{1 7})\end{array}$ & $\begin{array}{l}\text { Big } 12-2 \\
\quad(\mathbf{2 4})\end{array}$ & $\begin{array}{c}\text { Hybrid 12-2 } \\
(\mathbf{2 5})\end{array}$ \\
\hline \multirow{4}{*}{ Total } & beam $=1 \mathrm{bs}=1$ & 14464 & 6131 & 6535 & 6544 & 3519 \\
\hline & beam $=5 \mathrm{bs}=1$ & 23990 & 12646 & 13264 & 10873 & 7034 \\
\hline & beam $=1 \mathrm{bs}=64$ & 912 & 375 & 402 & 397 & 195 \\
\hline & \multirow{7}{*}{ beam $=5$ bs $=64$} & 1492 & 854 & 902 & 708 & 495 \\
\hline Encoder & & 21 & 21 & 41 & 41 & 41 \\
\hline Decoder & & 899 & 350 & 364 & 343 & 155 \\
\hline Self-attn / RNN & & 296 & 101 & 105 & 105 & 44 \\
\hline Cross-attn & & 257 & 85 & 89 & 88 & 42 \\
\hline Softmax & & 39 & 38 & 39 & 19 & 20 \\
\hline Beam top-k & & 207 & 204 & 209 & 40 & 40 \\
\hline
\end{tabular}

Table 19: Time benchmark across different ParaCrawl model sizes and decoding settings. Time in seconds spent decoding the concatenation of all $\rightarrow$ EN TED2020-valid sets (averages over 3 runs).

\begin{tabular}{cc|c|c|c|c} 
& Model & $\rightarrow$ EN & $\leftarrow$ EN & $/$ EN & WPS \\
\hline \multicolumn{5}{c}{ English-centric } \\
\hline $\mathbf{2}$ & Base 6-6 & $31.8 / 30.5$ & $24.2 / 23.6$ & $13.5 / 13.2$ & $724 / 385$ \\
$\mathbf{3}$ & Base 12-2 & $33.6 / 32.9$ & $24.3 / 24.2$ & $14.1 / 14.1$ & $1321 / 765$ \\
\hline \multicolumn{5}{c}{+ Multi-parallel } \\
\hline $\mathbf{5}$ & Base 6-6 & $32.8 / 31.5$ & $24.3 / 23.4$ & $16.3 / 15.6$ & $760 / 390$ \\
$\mathbf{6}$ & Base 12-2 & $33.5 / 32.7$ & $24.5 / 24.2$ & $16.3 / 16.0$ & $1258 / 723$ \\
$\mathbf{1 1}$ & Hybrid 12-2 & $32.8 / 31.7$ & $23.5 / 23.6$ & $16.1 / 15.6$ & $2546 / 1403$ \\
$\mathbf{1 2}$ & Hybrid 12-3 & $32.9 / 31.6$ & $23.7 / 23.6$ & $16.1 / 15.6$ & $2279 / 1338$
\end{tabular}

Table 20: TED Talks experiments on another MT framework show that our results are reproducible. The first number in each cell is the value obtained with fairseq and the second number is obtained with our internal TensorFlow implementation. Both implementations share the same hyper-parameters, with one notable exception: in the TensorFlow implementation, source/target embeddings are not shared. Additionally, the Hybrid models trained with fairseq use train-time BPE filtering, while the TensorFlow models do not. 

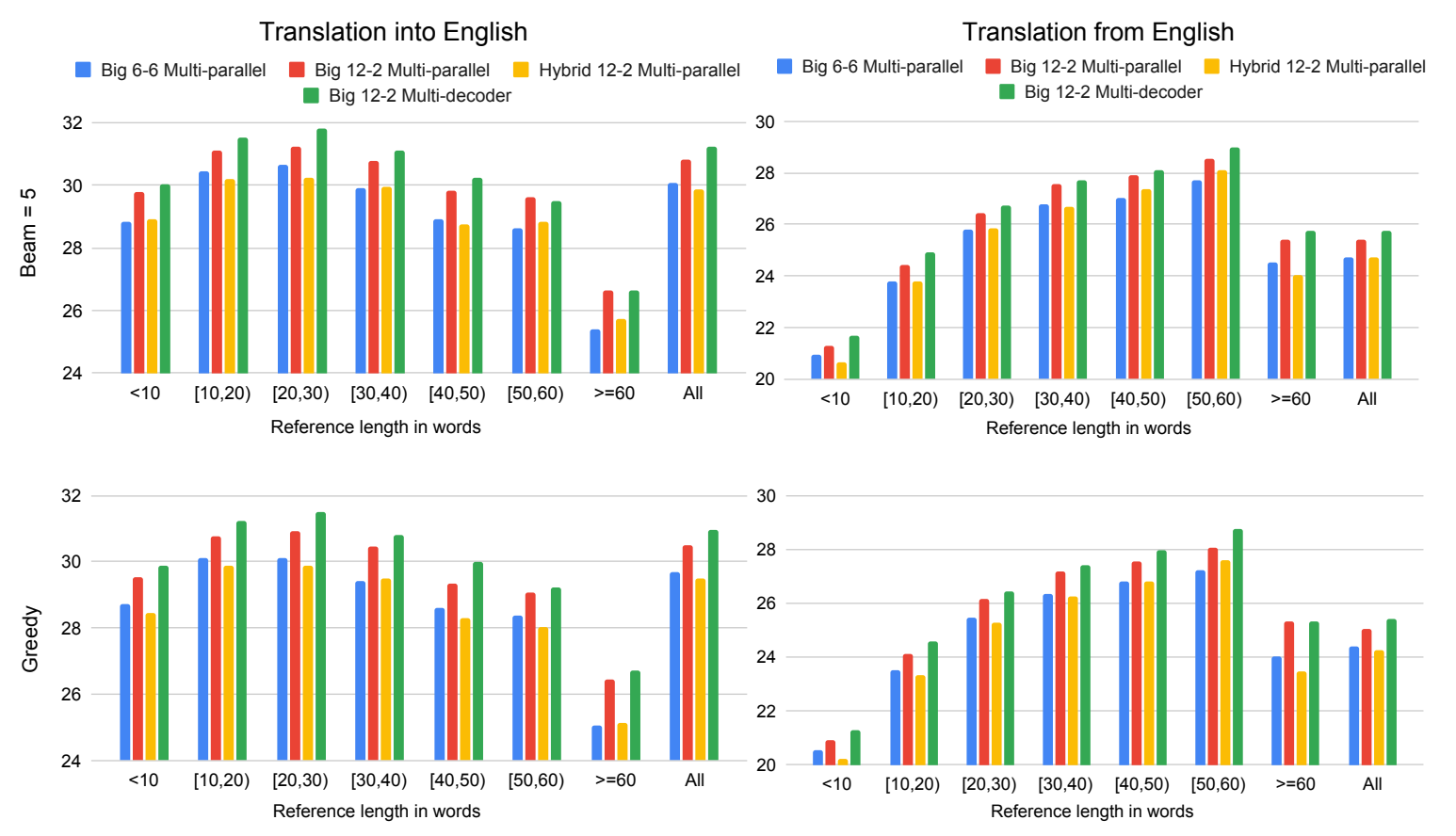

Figure 7: BLEU scores on $\leftrightarrow$ EN TED2020 test by the ParaCrawl models with beam search or greedy search (23, $\mathbf{2 4}, \mathbf{2 5}, \mathbf{2 8}$ ) according to sentence length. Top: beam search. Bottom: greedy search. Left: $\rightarrow$ EN translation. Right: $\leftarrow$ EN translation. 


\begin{tabular}{|c|c|c|c|c|c|c|c|c|}
\hline & Model & $\rightarrow \mathrm{EN}$ & Test BLEU & EU $/ \mathrm{EN}$ & \multicolumn{3}{|c|}{ Test chrF } & WPS \\
\hline \multicolumn{9}{|c|}{ SOTA (Philip et al., 2020) } \\
\hline & Bilingual & 32.4 & 24.4 & 15.0 & - & - & - & - \\
\hline & Best multi & 32.3 & 24.1 & 15.8 & - & - & - & - \\
\hline \multicolumn{9}{|c|}{ English-centric } \\
\hline 1 & Small 6-6 & 31.6 & 23.1 & $11.6(14.4)$ & .527 & .474 & $.342(.373)$ & 703 \\
\hline 2 & Base 6-6 & 31.8 & 24.2 & $13.5(15.0)$ & .529 & .486 & $.370(.381)$ & 753 \\
\hline 29 & $(2)+$ dec. lang code & 32.0 & 23.9 & $5.4(14.9)$ & .532 & .484 & $.186(.381)$ & 766 \\
\hline 36 & Base 6-2 & 32.5 & 23.3 & $12.7(14.6)$ & .536 & .475 & $.358(.377)$ & 1271 \\
\hline 3 & Base $12-2$, init with (2) & 33.6 & 24.3 & $14.1(15.4)$ & .548 & .487 & $.378(.389)$ & 1287 \\
\hline 33 & Base 12-2, pre-norm & 33.1 & 24.2 & $13.2(15.3)$ & .544 & .487 & $.368(.388)$ & 1312 \\
\hline 34 & Base $12-2$, enc. pre-norm & 33.3 & 24.3 & $12.7(15.3)$ & .546 & .487 & $.359(.389)$ & 1240 \\
\hline 30 & $(3)+$ dec. lang code & 33.4 & 22.8 & $0.7(14.7)$ & .547 & .472 & $.102(.380)$ & 1307 \\
\hline 38 & $(2)+$ TED-59 & 25.5 & 19.9 & $9.0(11.5)$ & .455 & .423 & $.282(.324)$ & 715 \\
\hline \multicolumn{9}{|c|}{+ Multi-parallel } \\
\hline 5 & Base 6-6 & 32.8 & 24.3 & 16.3 & .540 & .482 & .395 & 732 \\
\hline 39 & $(\mathbf{5})+K_{\text {test }}=10$ & 32.7 & 24.1 & 16.3 & .539 & .481 & .394 & 826 \\
\hline 40 & (5) $+N_{\text {test }}=4 \mathrm{k}$ & 30.8 & 22.3 & 15.2 & .519 & .463 & .382 & 868 \\
\hline 6 & Base 12-2 & 33.5 & 24.5 & 16.3 & .546 & .485 & .395 & 1203 \\
\hline 7 & $(\mathbf{6})+K_{\text {test }}=10$ & 33.4 & 24.3 & 16.2 & .545 & .484 & .394 & 1539 \\
\hline 8 & $(\mathbf{6})+N_{\text {test }}=4 \mathrm{k}$ & 31.5 & 22.5 & 15.2 & .523 & .466 & .382 & 1457 \\
\hline 41 & Hybrid 12-2 & 32.9 & 23.7 & 16.2 & .544 & .476 & .394 & 1724 \\
\hline 42 & Hybrid 12-3 & 32.9 & 23.8 & 16.2 & .543 & .476 & .395 & 1533 \\
\hline \multicolumn{9}{|c|}{+ BPE filtering $\left(N_{\text {train }}=4 \mathrm{k}\right)$} \\
\hline 9 & Base 6-6 & 32.9 & 24.2 & 16.3 & .541 & .481 & .394 & 789 \\
\hline 43 & $(9)+$ dec. lang code & 32.7 & 23.9 & 16.2 & .540 & .478 & .393 & 891 \\
\hline 44 & Base 6-2 & 32.5 & 23.4 & 15.6 & .537 & .472 & .386 & 1595 \\
\hline 10 & Base 12-2 & 33.3 & 24.3 & 16.3 & .546 & .481 & .395 & 1552 \\
\hline 45 & $(\mathbf{1 0})+$ dec. lang code & 32.5 & 23.0 & 15.7 & .540 & .471 & .389 & 1514 \\
\hline 11 & Hybrid 12-2 & 32.8 & 23.5 & 16.1 & .543 & .474 & .393 & 2422 \\
\hline 12 & Hybrid $12-3$ & 32.9 & 23.7 & 16.1 & .543 & .475 & .394 & 2145 \\
\hline \multicolumn{9}{|c|}{ + Multi-decoder } \\
\hline 13 & $(2) \rightarrow 6-2$ & 33.0 & 24.2 & 16.0 & .543 & .480 & .391 & 1608 \\
\hline 46 & $(36) \rightarrow 6-2$ & 33.3 & 24.5 & 16.2 & .545 & .482 & .394 & 1548 \\
\hline 14 & (3) $\rightarrow 12-2$ & 33.8 & 25.1 & 16.7 & .551 & .490 & .401 & 1614 \\
\hline 47 & (14) w/o lang code & 33.6 & 24.3 & 16.6 & .550 & .481 & .399 & 1567 \\
\hline
\end{tabular}

Table 21: Test BLEU, chrF scores and decoding speed of TED Talks models of various depths. SOTA's "best multi" is a Transformer Small 6-6 multi-parallel model with adapter layers. WPS: speed in words per second for $\rightarrow$ EN translation. Scores in parentheses are obtained by pivot translation through English. (47) does not use any language code during the multi-decoder finetuning stage. 


\begin{tabular}{|c|c|c|c|c|c|c|c|c|}
\hline & \multirow{2}{*}{ Model } & \multicolumn{3}{|c|}{ TED2020 test chrF } & \multicolumn{3}{|c|}{ FLORES devtest spBLEU } & \multirow{2}{*}{ WPS } \\
\hline & & $\rightarrow \mathrm{EN}$ & $\leftarrow \mathrm{EN}$ & / EN & $\rightarrow \mathrm{EN}$ & $\leftarrow \mathrm{EN}$ & / EN & \\
\hline & Goyal et al. (2021) & - & - & - & 32.4 & 31.9 & 25.7 & - \\
\hline & \multicolumn{8}{|c|}{ English-centric } \\
\hline 15 & Base 6-6 & .551 & .545 & $.399(.456)$ & 34.0 & 31.9 & $16.0(22.5)$ & 656 \\
\hline 16 & Big 6-6 & .580 & .569 & $.401(.483)$ & 38.8 & 36.4 & $18.5(27.0)$ & 623 \\
\hline 37 & Big 6-2 & .572 & .565 & $.373(.477)$ & 37.7 & 35.3 & $15.3(25.8)$ & 1077 \\
\hline 17 & Big 12-2 & .585 & .575 & $.435(.488)$ & 39.6 & 37.1 & $21.1(27.6)$ & 1033 \\
\hline 48 & Wide $12-2$ & .590 & .581 & $.381(.494)$ & 40.7 & 38.9 & $19.0(\mathbf{2 9 . 0})$ & 870 \\
\hline \multicolumn{9}{|c|}{ + Multi-parallel } \\
\hline 19 & Big 6-6 & .574 & .564 & .481 & 37.9 & 35.6 & 26.8 & 595 \\
\hline 49 & Big 6-6 $(T=5)$ & .573 & .564 & .481 & 37.6 & 35.6 & 26.8 & 608 \\
\hline 20 & Big 12-2 & .581 & .570 & .486 & 39.0 & 36.2 & 27.6 & 1030 \\
\hline 21 & $(\mathbf{2 0})+N_{\text {test }}=16 \mathrm{k}$ & .580 & .570 & .486 & 38.4 & 35.8 & 27.2 & 1328 \\
\hline 22 & $(\mathbf{2 0})+N_{t e s t}=8 \mathrm{k}$ & .563 & .560 & .478 & 33.5 & 33.4 & 25.3 & 1283 \\
\hline 50 & Hybrid 12-3 & .576 & .564 & .482 & 38.2 & 35.4 & 26.9 & 1313 \\
\hline \multicolumn{9}{|c|}{+ BPE filtering $\left(N_{\text {train }}=8 \mathrm{k}\right)$} \\
\hline 23 & Big 6-6 & .572 & .564 & .480 & 37.5 & 35.5 & 26.6 & 679 \\
\hline 51 & Big 6-2 & .565 & .558 & .472 & 36.2 & 34.0 & 25.1 & 1305 \\
\hline 24 & Big 12-2 & .580 & .570 & .486 & 38.8 & 36.2 & 27.4 & 1261 \\
\hline 25 & Hybrid 12-2 & .573 & .562 & .481 & 37.9 & 34.9 & 26.5 & 1796 \\
\hline 52 & $(\mathbf{2 5})+200 \mathrm{k}$ steps & .576 & .564 & 483 & 38.2 & 34.9 & 26.8 & 1861 \\
\hline 26 & Hybrid $12-3$ & .574 & .563 & .482 & 38.0 & 34.8 & 26.6 & 1659 \\
\hline 53 & $(\mathbf{2 6})+200 \mathrm{k}$ steps & .577 & .565 & .483 & 38.3 & 35.6 & 27.1 & 1770 \\
\hline \multicolumn{9}{|c|}{ + Multi-decoder } \\
\hline 27 & $(\mathbf{1 6}) \rightarrow 6-2$ & .573 & .566 & .483 & 38.0 & 36.3 & 27.3 & 1333 \\
\hline 54 & $(27)+200 \mathrm{k}$ steps & .575 & .569 & 484 & 38.1 & 36.7 & 27.5 & 1360 \\
\hline 55 & $(\mathbf{2 3}) \rightarrow 6-2$ & .573 & .566 & .483 & 37.9 & 36.4 & 27.3 & 1372 \\
\hline 56 & $(37) \rightarrow 6-2$ & .574 & .566 & .483 & 38.0 & 36.2 & 27.4 & 1295 \\
\hline 57 & $(\mathbf{5 1}) \rightarrow 6-2$ & .574 & .567 & .484 & 37.9 & 36.1 & 27.4 & 1323 \\
\hline 28 & $(\mathbf{1 7}) \rightarrow 12-2$ & .583 & .573 & .490 & 39.5 & 37.4 & 28.7 & 1270 \\
\hline 58 & $(\mathbf{2 8})+200 \mathrm{k}$ steps & .585 & .574 & .492 & 39.8 & 37.5 & 28.9 & 1221 \\
\hline 59 & $(\mathbf{2 4}) \rightarrow 12-2$ & .583 & .572 & .491 & 39.5 & 37.2 & 28.6 & 1274 \\
\hline 60 & $(\mathbf{2 8})+$ freeze enc. & .584 & .574 & .478 & 39.5 & 37.5 & 26.5 & 1238 \\
\hline 61 & (59) + freeze enc. & .581 & .574 & .489 & 39.2 & 37.3 & 28.3 & 1219 \\
\hline 62 & (28) w/o lang code & .583 & .573 & .491 & 39.3 & 37.3 & 28.6 & 1215 \\
\hline 63 & $(\mathbf{6 2})+200 \mathrm{k}$ steps & .585 & .575 & .493 & 39.7 & 37.7 & 29.0 & 1242 \\
\hline 64 & $(\mathbf{2 8})+$ shared embed & .582 & .572 & .489 & 39.3 & 37.2 & 28.4 & 1224 \\
\hline
\end{tabular}

Table 22: TED2020 test chrF, FLORES devtest spBLEU (Goyal et al., 2021) and decoding speed of ParaCrawl models of various depths. WPS: speed in words per second for $\rightarrow E N$ translation. Scores in parentheses are obtained by pivot translation through English. (48) uses the same architecture as (17) but with a feed-forward dimension of 8192 and is trained for 1M steps on ParaCrawl v8. (62) is fine-tuned like (28) but without language codes, similarly to Lyu et al. (2020) (while the pre-trained model has language codes). (60, 61) freeze the shared encoder parameters during the multi-decoder training stage. (64) is trained with shared target embeddings and $N_{\text {train }}=8 \mathrm{k}$ (instead of training one separate embedding matrix per decoder). 